\title{
Webs and posets
}

\author{
M. Dukes, ${ }^{a}$ E. Gardi, ${ }^{b}$ H. McAslan, ${ }^{c}$ D.J. Scott ${ }^{d}$ and C.D. White ${ }^{e}$ \\ ${ }^{a}$ Department of Computer and Information Sciences, University of Strathclyde, \\ Glasgow G1 $1 X H$, U.K. \\ ${ }^{b}$ Higgs Centre for Theoretical Physics, School of Physics and Astronomy, \\ The University of Edinburgh, \\ Edinburgh EH9 3JZ, Scotland, U.K. \\ ${ }^{c}$ Department of Physics and Astronomy, University of Sussex, \\ Brighton BN1 9QH, U.K. \\ ${ }^{d}$ Institute for Particle Physics Phenomenology, University of Durham, \\ Durham DH1 3LE, U.K. \\ e SUPA, School of Physics and Astronomy, University of Glasgow, \\ University Avenue, Glasgow G12 8QQ, Scotland, U.K. \\ E-mail: mark.dukes@strath.ac.uk, Einan.Gardi@ed.ac.uk, \\ H.Mcaslan@sussex.ac.uk, d.j.scott@durham.ac.uk, \\ Christopher. White@glasgow.ac.uk
}

ABstRACT: The non-Abelian exponentiation theorem has recently been generalised to correlators of multiple Wilson line operators. The perturbative expansions of these correlators exponentiate in terms of sets of diagrams called webs, which together give rise to colour factors corresponding to connected graphs. The colour and kinematic degrees of freedom of individual diagrams in a web are entangled by mixing matrices of purely combinatorial origin. In this paper we relate the combinatorial study of these matrices to properties of partially ordered sets (posets), and hence obtain explicit solutions for certain families of web-mixing matrix, at arbitrary order in perturbation theory. We also provide a general expression for the rank of a general class of mixing matrices, which governs the number of independent colour factors arising from such webs. Finally, we use the poset language to examine a previously conjectured sum rule for the columns of web-mixing matrices which governs the cancellation of the leading subdivergences between diagrams in the web. Our results, when combined with parallel developments in the evaluation of kinematic integrals, offer new insights into the all-order structure of infrared singularities in non-Abelian gauge theories.

KeYwords: QCD, Scattering Amplitudes, Resummation

ARXIV EPRINT: 1310.3127 


\section{Contents}

1 Introduction 1

2 Webs and web-mixing matrices 4

3 The rank of web-mixing matrices $\quad 9$

$\begin{array}{lll}4 & \text { Webs and posets } & 13\end{array}$

5 General solutions for web-mixing matrices $\quad \mathbf{1 5}$

5.1 The $(1,2,2, \ldots, 2,1)$ web 16

5.2 The $(1,1,1, \ldots, 1, n)$ web 23

6 The weighted column sum rule $\quad 26$

$6.1 s(D)$ and linear extensions $\quad 27$

6.2 Column sum rule for $(1,1, \ldots, 1, n)$ webs $\quad 27$

6.3 Column sum rule for $(1,2, \ldots, 2,1)$ webs 28

$\begin{array}{lll}7 & \text { Conclusion } & 30\end{array}$

A Examples of web-mixing matrices $\quad 32$

A.1 $(1,2,2, \ldots 2,1)$ webs 32

A.2 $(1,1,1, \ldots 1, n)$ webs 33

$\begin{array}{ll}\text { B Eulerian numbers } & 34\end{array}$

C Results for $s(D)$ for $(1,2,2, \ldots, 2,1)$ webs 35

\section{Introduction}

Wilson lines continue to generate significant amounts of interest, due to their role in a variety of phenomenological and theoretical applications [1-32]. For example, they govern the structure of infrared singularities in scattering amplitudes, ${ }^{1}$ which lead to large kinematic logarithms which have to be summed up to all orders in perturbation theory [15-17, 34-55]. They also govern scattering in the high energy limit in both gauge theories and gravity [5672]. For these and other applications, it is important to classify the behaviour of correlators of products of Wilson line operators. Whilst much is known about the case of two Wilson lines meeting at a cusp [73-75], the general case of more than two Wilson lines has been studied only recently. The anomalous dimension has been calculated up to two-loop order

\footnotetext{
${ }^{1}$ For a pedagogical review, see [33].
} 
for both massless $[76,77]$ and massive [78-80] particles, where in the former case it was found to have the same colour structure as the one-loop result. This was later explained by constraints on the structure of infrared singularities in massless scattering amplitudes which follow from factorisation and rescaling symmetry, leading to the formulation of the dipole formula [81-83]), a minimal all-order ansatz for the anomalous dimension. Possible corrections to this form have been investigated further in refs. [66, 67, 84-87]. Further progress in this area requires explicit calculations of Wilson line correlators at higher loop orders. In practice this means calculating the exponents of correlators of Wilson lines, from which the relevant anomalous dimensions can be extracted. This itself necessitates the development of efficient computational techniques.

In a recent series of papers [88-91] (see also [92]), a diagrammatic method has been developed, which allows us to calculate the exponents of Wilson line correlators directly. This provides an efficient framework in which to address higher loop contributions to the multileg soft anomalous dimension, and is a generalisation of the well-known diagrammatic exponentiation of correlators of two Wilson lines (or a Wilson loop) in terms of webs [7375]. As is explained in detail in ref. [88], however, this generalisation is highly non-trivial. Whereas webs in two-parton scattering are single irreducible diagrams, which are each individually free of subdivergences associated with renormalisation of the cusp, in multi-parton scattering webs are closed sets of diagrams related by permutations of gluon emissions on the Wilson lines (we review this material in more detail in section 2). The general form of a given correlator of Wilson line operators $\Phi_{i}$ is then given by

$$
\left\langle\prod_{i=1}^{L} \Phi_{i}\right\rangle=\exp \left[\sum_{W} W\right],
$$

where the contribution of each web is

$$
W=\sum_{D, D^{\prime}} \mathcal{F}(D) R_{D D^{\prime}} C\left(D^{\prime}\right)
$$

Here the sums are over all diagrams $D, D^{\prime}$ in the web, whose kinematic and colour parts are given by $\mathcal{F}(D)$ and $C\left(D^{\prime}\right)$ respectively. The matrix $R_{D D^{\prime}}$ consists of rational numbers of combinatorial origin, and acts to entangle the colour and kinematic degrees of freedom. Each web has its own web-mixing matrix, and these are known to have special properties. Chief among these is idempotence (proven in ref. [89]), which implies that web mixing matrices are projection operators, with eigenvalues in the set $\{0,1\}$. Each unit eigenvalue is associated with a combination of kinematic factors which survives in the exponent, and which has an associated exponentiated colour factor (ECF), consisting of a superposition of colour factors of individual diagrams in the web. It has recently been shown that all such ECFs correspond to colour factors of diagrams in which all gluons are connected (once the Wilson lines have been removed), thus generalising the so-called non-Abelian exponentiation theorem from the two-line to the multiline case [91].

Other properties of web-mixing matrices are known, such as the fact that their rows sum to zero $[88,89]$, and that their columns appear to sum to zero after contraction with 
appropriate weight factors [90]. These features are related to two underlying principles: (a) colour factors in the exponent must be fully connected (as described above); (b) the corresponding combinations of kinematic factors are only allowed to contain specific higherorder divergences, which can be cancelled by highly constrained subtraction terms involving subdiagrams of the given web (see [90]). It is therefore clear that a deeper understanding of the combinatorics of web-mixing matrices can provide a valulable insight into the structure of infrared singularities in non-Abelian gauge theories, to all orders in perturbation theory. This is only part of the story, however. A full understanding of higher order singularity structures requires calculation of the necessary kinematic integrals. To this end, new computational techniques are becoming available, which offer the possibility of calculating certain families of kinematic integral to any order in perturbation theory [93, 94]. Clearly, analysis of the structure of web mixing matrices and that of kinematic integrals should proceed in parallel.

The aim of this paper is to investigate the combinatorial structure of web mixing matrices in more detail. We will present a useful classification of webs based on the number of external lines they connect before focusing on the special case of webs which connect $L+1$ lines with $L$ single gluon exchanges. We will present a simple formula for the rank of any web-mixing matrix in this class and, thus, for the number of independent connected colour factors such a web contributes to. We will then examine the results of ref. [95], a recent study which considered web-mixing matrices from a pure mathematical point of view, and which related their combinatorics to that of order-preserving maps on partially ordered sets (posets), for which a large mathematical literature exists. Using the poset language, we will be able to completely solve for the web-mixing matrices for two special families of webs, at arbitrary order in perturbation theory. The calculation of the corresponding kinematic integrals will be considered elsewhere [93, 94]. Finally, we will interpret a recently conjectured column sum rule for web-mixing matrices [90] in the poset language, and look at this in detail for the two particular web families whose web-mixing matrices we are able to exactly solve for.

Although much about web-mixing matrices and their associated kinematic combinations remains unexplored (particularly for webs which contain three and four-gluon vertices away from the Wilson lines), their classification through combinatorial methods offers new insights into the structure of non-Abelian gauge theory. The web-mixing matrix solutions we present here correspond to all-order properties in the exponents of scattering amplitudes, thus correspond to very deep structures in perturbation theory. There is a clear motivation for pushing such investigations to broader web families, in tandem with the calculation of the relevant kinematic integrals.

The structure of the paper is as follows. In section 2 we review the definition of web-mixing matrices, in a self-contained fashion. In particular, we give a combinatorial definition of web mixing matrix elements (first presented in ref. [89]) that will be used frequently throughout the rest of the paper. In section 3 we consider how to systematically classify webs, and provide an expression for the rank of a particular class of webs, as mentioned above. In section 4, we introduce terms and properties relating to posets, which will not necessarily be familiar to a particle physics audience. In section 5 we consider 


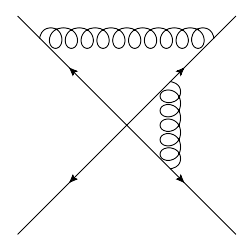

(a)

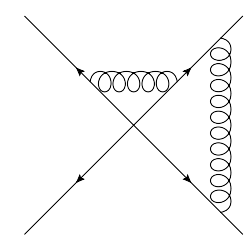

(b)

Figure 1. A $(1,2,1)$ web, connecting three parton lines at two-loop order.

two particular classes of webs, and use the poset language to obtain explicit solutions for their associated web-mixing matrices, before verifying that they encode fully connected colour factors. In section 6 we discuss the interpretation of the weighted column sum rule of ref. [89] in terms of linear extensions, and verify that this holds for the two web families considered in section 5 . In section 7 we discuss our results and conclude.

\section{Webs and web-mixing matrices}

The aim of this section is to briefly review previous results on webs and their mixing matrices, which will be useful for the rest of the paper. Note that different conventions exist in the literature for what is meant by a multiparton web [88, 92] (see in particular appendix A of ref. [89] for a discussion of this point). Throughout this paper, we adopt the terminology of refs. [88-91].

A multiparton web is a set of diagrams contributing to the exponent of a vacuum expectation value of Wilson line operators meeting at a point (alternatively, to the soft gluon part of a QCD scattering amplitude). An example is shown in figure 1, which illustrates a web connecting three Wilson lines (out of a total of four) at two-loop order. This consists of a pair of diagrams, which are related by permutations of gluon attachments on the external lines. Furthermore, the set is closed under such permutations: permuting the gluons in diagram (a) leads to diagram (b), and vice versa. In general, a web is any such set of diagrams closed under permutations of gluon attachments. Note that the two-loop web of figure 1 consists of distinct gluon exchanges. However, there could also be three and four-gluon vertices off the eikonal lines. This does not affect our definition of a web, which only concerns permutations of gluon attachments associated with the hard external (Wilson) lines. Many examples of webs can be found in refs. [88-91], and we will see more throughout the present paper. Note that any diagram that consists of a single connected piece (when the Wilson lines are removed) is a web by itself; we will not be interested in such webs in this paper as they do not present any combinatorial problem.

We may label a given web using a notation $\left(n_{1}, n_{2} \ldots n_{L}\right)$, where $n_{i} \geq 0$ is the number of gluon attachments on parton line $i$, and it is assumed that there are $L$ lines in total. Note that this notation does not uniquely specify a given web. In particular, it does not specify possible attachments of gluons off the eikonal lines, via three and four-gluon vertices. This will nevertheless be a convenient notation in what follows, since our interest here is in multiple gluon exchange diagrams with no three or four gluon vertices. 

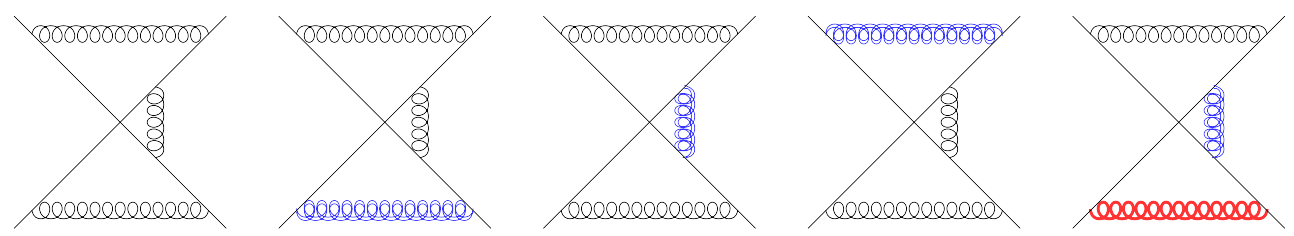

Figure 2. The partitions of the diagram on the far left-hand side, with $m=1,2,2,2,3$ respectively. Note that we have used different symbols to distinguish gluons belonging to different partition elements (double line, thick line), in addition to colours.

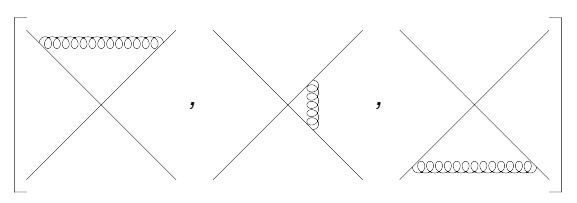

Figure 3. Alternative notation for the 3-partition of figure 2, in which each element of the partition is represented by its own diagram.

Given a web $W$, we may consider all diagrams $D \in W$. Each diagram $D$ has a kinematic part $\mathcal{F}(D)$ and a colour factor $C(D)$. Then the contribution of $W$ to the exponent of the Wilson line correlator is given by eq. (1.2), where $R_{D D^{\prime}}$ is a web-mixing matrix. The existence of web-mixing matrices was first derived using statistical physics methods (the "replica trick"), which also provides an algorithm for how to calculate them [88]. One may also provide a closed-form combinatorial formula for the element $R_{D D^{\prime}}$. To this end, one needs the notion of a partition of a web diagram $D \in W$. An m-partition of diagram $D$ is a colouring of $D$ with a number of distinct colours $m \leq n_{c}$, where $n_{c}$ is the number of connected subdiagrams ${ }^{2}$ in $D$. This is illustrated in figure 2 , which shows an example web diagram at three-loop order together with its partitions (note that the diagram itself is an $m$-partition with $m=1$ ). Although thinking of partitions as colourings gives an easy way to represent them, we actually use another notation to represent partitions: an $m$-partition of a diagram $D$ will be represented as a set with $m$ elements, each of which consists of the usual diagram notation, but only including gluons of a particular colour. To illustrate this, consider the 3-partition on the far right of figure 2. We may represent this using the alternative notation of figure 3. Here, each separate colour in the 3-partition gets its own diagram, and there are thus 3 elements in the set. Furthermore, we can then dispense with the colours altogether, as these are no longer needed to separate out the elements of the partition.

The reason for using this alternative notation for partitions is that the diagrams are subject to a multiplication rule, which we have illustrated in figure 4 . The rule for combining diagrams is as follows: to form the product, one takes the gluon part of each diagram, and draws them successively emanating outwards from the center of the product diagram. In figure 4(a), for example, the gluon joining external lines 1 and 2 (counting clockwise from top-left) appears closer to the center than the gluon joining external lines 2 and 3 in the product. Another example is shown in figure 4(b). Here the first diagram on the

\footnotetext{
${ }^{2}$ By a connected subdiagram, we mean a part of the diagram that forms a single connected piece, if the external (Wilson) lines have been removed.
} 

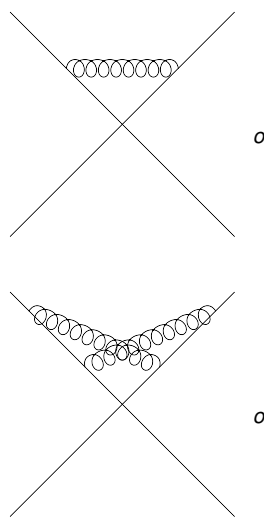

(1)

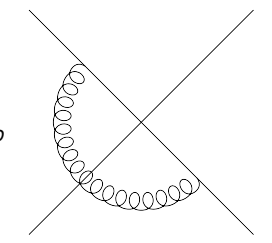

(c)
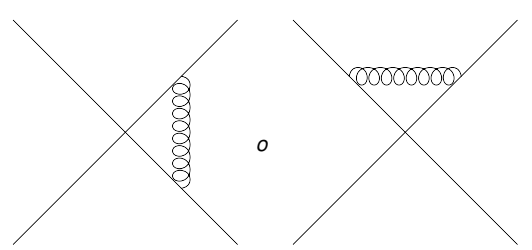
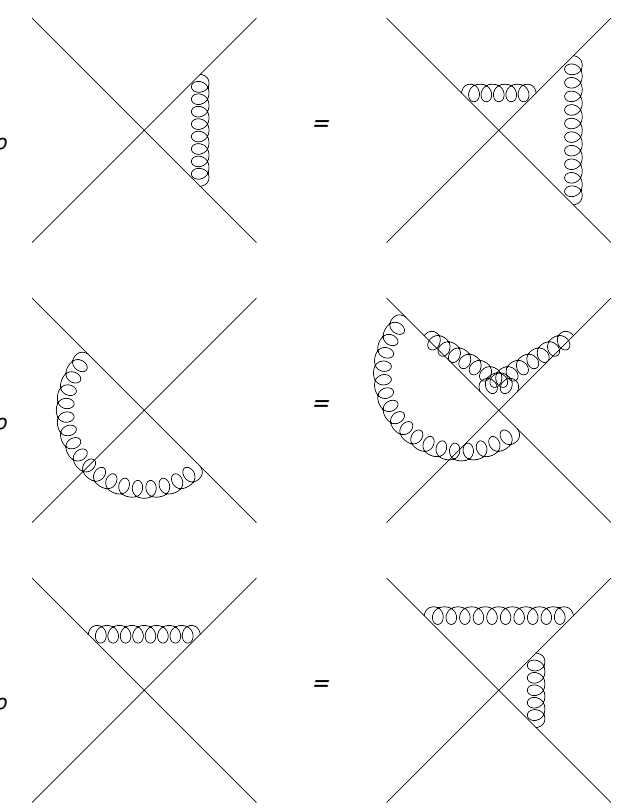

Figure 4. Examples of the multiplication rule $D_{1} \circ D_{2}$ for diagrams.

left-hand side itself has two gluons. In the product diagram, this entire structure is reproduced, followed by the gluon which joins lines 1 and 3. One may also think of this rule as operating separately on the external lines: on each external line, one must order the gluon attachments outwards from the center of the diagram, according to the order in which they appear in the product of individual diagrams on the left-hand side. The reason for considering this multiplication rule is that it describes how the colour factor of a composite diagram is formed from the colour factors of its constitutents. That is, denoting the product of diagrams $D_{1}$ and $D_{2}$ via $D_{1} \circ D_{2}$, the colour factor of the product is given by

$$
C\left(D_{1} \circ D_{2}\right)=C\left(D_{1}\right) C\left(D_{2}\right) \text {. }
$$

Note that this multiplication rule for diagrams is non-commutative, corresponding to the fact that colour matrices associated with gluon emissions are themselves non-commuting. For example, the alternative ordering of the diagrams in figure 4(a) gives the product shown in figure 4(c), which is indeed not the same. Rather, it is a different diagram belonging to the same web.

Having defined the above multiplication rule, we can apply it within $m$-partitions of a given diagram, by multiplying the elements together. A given $m$-partition can be used to make $m$ ! diagrams (where all elements of the partition are used in each product), according to the number of permutations of the elements. Note that some of the diagrams one forms may be the same. Consider, for example, the 3-partition from figure 3. This can be used to make 6 diagrams, which must belong to the web shown in figure 5 . Labelling the elements of figure 3 by $[1,2,3]$, the diagrams that result from each permutation are shown in figure 6. Comparing this with figure 5, we see that diagrams (a) and (d) are both made twice, whereas diagrams (b) and (c) are made once only. The sum of these values 


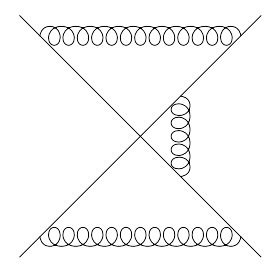

(a)

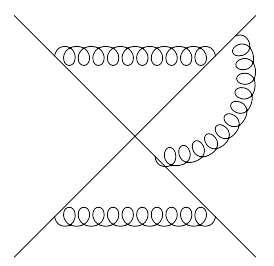

(b)

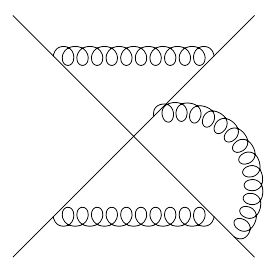

(c)

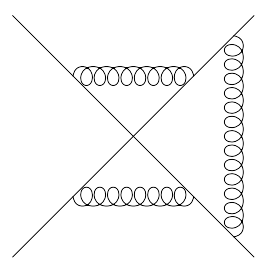

(d)

Figure 5. Three-loop web containing the diagram of figure 2 .

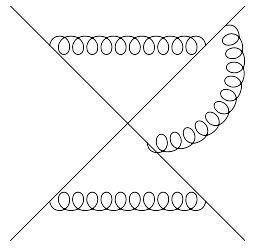

(123)

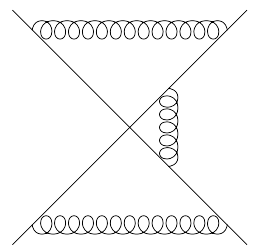

(231)

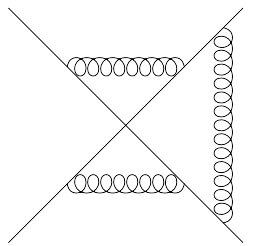

(132)

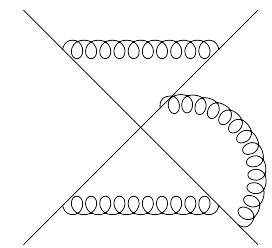

(321)

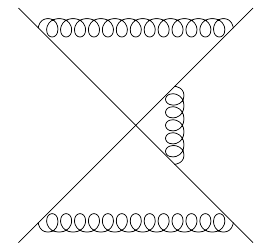

(213)

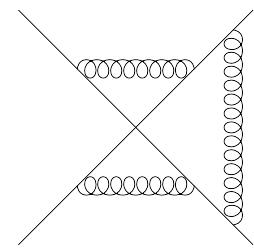

(312)

Figure 6. Diagrams formed by multiplying all permutations of elements in the 3-partition of figure 3 .

is $2+2+1+1=3$ ! as required. One may carry out a similar exercise for any $m$-partition of any diagram, and this leads us to define the following quantity, first introduced in ref. [89]:

The "overlap function of $D^{\prime}$ with respect to $P_{D}$ ", denoted by $\left\langle D^{\prime} \mid P_{D}\right\rangle$, is the number of ways that diagram $D^{\prime}$ is made upon multiplying together all permutations of the elements of the partition $P_{D}$ of diagram $D$. Here $D$ and $D^{\prime}$ are diagrams in the same closed set (related by gluon permutations).

In the example of figure 6, one finds $\langle d \mid P\rangle=\langle a \mid P\rangle=2$ and $\langle b \mid P\rangle=\langle c \mid P\rangle=1$, where $P$ is the 3-partition of diagram (a), shown in figure 3 . Note that, in general, one has

$$
\sum_{D^{\prime}}\left\langle D^{\prime} \mid P_{D}\right\rangle=n\left(P_{D}\right) !
$$

for any fixed partition $P_{D}$, where $n\left(P_{D}\right)$ is the number of elements in this partition. That is, every permutation of the elements of $P_{D}$ makes some diagram in the set to which $D$ and $D^{\prime}$ belong. Thus, summing over the overlap functions, by their definition, leads to the total number of permutations of the elements, which is $n\left(P_{D}\right)$ !.

Given the notion of an overlap function, one may show that the web mixing-matrix element $R_{D D^{\prime}}$ is given by [89]

$$
R_{D D^{\prime}}=\sum_{P_{D}} \frac{(-1)^{n\left(P_{D}\right)-1}}{n\left(P_{D}\right)}\left\langle D^{\prime} \mid P_{D}\right\rangle .
$$


There is a sum over all partitions $P_{D}$ of a given diagram $D$ on the right-hand side, such that the left-hand side still depends on $D$ (and indeed on $D^{\prime}$ which appears in the overlap function in each term on the right-hand side). As already discussed in the introduction, this formula is purely combinatorial in nature, and forms a basis for the formal study of web-mixing matrices [95].

Some general properties of web-mixing matrices have already been established. Firstly, all web-mixing matrices are idempotent:

$$
\sum_{E} R_{D E} R_{E D^{\prime}}=R_{D D^{\prime}}
$$

This means that web-mixing matrices are projection operators, whose eigenvalues are 0 and 1 (with associated multiplicities). This fact has been proven using statistical physics methods [89], but a combinatorial proof remains elusive. The physical interpretation of the idempotence property was examined in ref. [88] and then in detail in refs. [90, 91]. Unit eigenvalues of a web-mixing matrix are associated with certain combinations of kinematic factors, each of which has an accompanying colour factor which corresponds to that of a fully connected gluon diagram [91]. Note in particular that the idempotence property implies that the rank of any web-mixing matrix is the same as its trace. The physical meaning of the rank is that it specifies the number of independent connected colour factors to which a given web contributes.

A second known property is that the row sums of web-mixing matrices are always zero:

$$
\sum_{D^{\prime}} R_{D D^{\prime}}=0 \quad \forall D
$$

This was proven using combinatorial methods in ref. [89], and this property expresses the fact that the fully symmetric colour factor that can be obtained from a given web does not contribute to the exponent of the amplitude. Rather, it is generated in the amplitude itself by the exponentiation of lower-order contributions. Also in ref. [89], the sum rule was generalised for planar and non-planar subsets of diagrams in a given web. This provides an interesting link between the combinatorial properties of web-mixing matrices, and the $1 / N_{c}$ expansion, where $N_{c}$ is the number of colours.

Thirdly, all web-mixing matrices are conjectured to obey the weighted column sum rule:

$$
\sum_{D} s(D) R_{D D^{\prime}}=0 \quad \forall D^{\prime}
$$

where $s(D)$ is the number of ways of independently shrinking connected subdiagrams to the origin, and such that $s(D)=0$ for diagrams that are not maximally reducible. For example, in the diagrams of figure 5(b) and (c), there is only one way in each graph of shrinking all three gluons independently to the origin, whereas in (a) and (d), there are two ways, depending on whether one shrinks the upper or lower gluon first. Thus, in this case the vector of multiplicity factors is given by $(2,1,1,2)$. More examples of the $s(D)$ factors were presented in ref. [89], and a justification of the column conjecture given for graphs which do not contain three or four gluon vertices. The physics of this result concerns 
the renormalisation of the hard interaction vertex at which the Wilson lines meet, and the physical justification of eq. (2.6) relied upon the fact that individual webs renormalise independently, with no mixing between webs. A combinatorial proof of eq. (2.6) would provide a basis for generalising the column sum rule, analogous to the sub-row sum rule introduced in ref. [89]. This would provide a link between subleading kinematic divergences, and subleading terms in the $1 / N_{c}$ expansion, as hinted at above. We will return later in the paper to the combinatorial problem of determining the vector $s(D)$ for certain webs, and also see how the weighted column sum rule can be obtained combinatorially in particular cases.

In this section, we have reviewed a number of ideas relating to web mixing matrices, which pave the way for further combinatorial study of their general properties. In the following section, we begin such an investigation, by examining the rank of web-mixing matrices in particular cases.

\section{The rank of web-mixing matrices}

Our aim in this section is provide a simple formula for the rank of a given class of webmixing matrices, and in doing so to provide a systematic classification of webs that is convenient for further study. As explained in the previous section, the rank of a web-mixing matrix has a simple physical meaning: it dictates how many independent connected colour factors a given web contributes to in the exponent of a Wilson line correlator. The general programme for calculating this exponent at a given order in perturbation theory is as follows [91]:

1. Pick a basis of connected colour factors, sufficient to span the space of all possible colour factors. This basis is not unique e.g. an alternative choice of colour factors can be generated by applying Jacobi identities. Reference [91] introduced an effective vertex formalism for calculating web graphs, which gave a convenient prescription for choosing a basis of colour factors.

2. Once a basis has been chosen, one must consider all webs at the given perturbative order. For each web, one must ascertain which connected colour factors it contributes to, and work out what the corresponding combinations of kinematic factors are that accompany each colour factor. This can be done either using web-mixing matrices, whose eigenvectors of unit eigenvalue are in one-to-one correspondence with connected colour factors, or by using the effective vertex formalism discussed in ref. [91].

3. The total contribution to a given colour factor can now be calculated, by carrying out the kinematic integrals found in the previous step. This will be a gauge invariant quantity, consisting of contributions from all webs which contribute to the given colour factor. We call such a quantity a gauge-invariant web, and its calculation may benefit from the use of cleverly designed gauges such as that presented in ref. [24].

A current priority is to calculate the relevant gauge-invariant web combinations at threeloop order, which will extend the state of the art of present-day resummation techniques by a further logarithmic order, leading to significant improvements in the precision of collider 

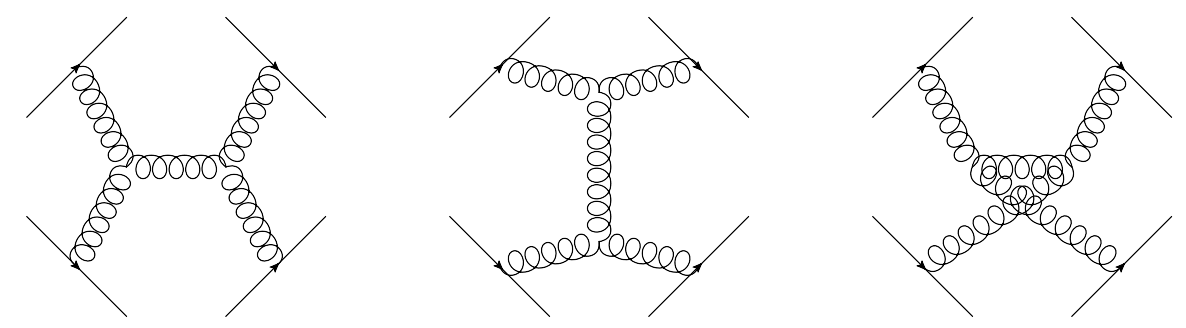

Figure 7. The three possible connected colour factors connecting four Wilson lines at three-loop order.

physics predictions. To this end, all required combinations of kinematic factors have been presented, for a given colour factor basis, in ref. [91]. Calculation of the kinematic integrals is also underway [93, 94], and involves the use of techniques that potentially generalise to higher orders in perturbation theory. This leads to the exciting possibility that one could calculate kinematic factors for given web families for any number of gluons, which strongly motivates seeking an all-order elucidation of web properties. One of the simpler - and most useful - properties to consider is the rank of web-mixing matrices. A general understanding of which connected colour factors come from which webs is a prerequisite for constructing gauge-invariant webs at a given perturbative order, and the rank provides a first step in this regard.

In investigating the above questions, it is convenient to classify webs according to how many Wilson lines they connect, as was already done in the three-loop analysis of ref. [91]. In general, webs may connect $m$ lines at $n$-loop order, where $1 \leq m \leq n+1$. The case of $m=1$ consists of pure self-energy like contributions, which vanish for massless external lines. The case of $m=2$ consists of diagrams which also enter the calculation of the traditional (two-loop) cusp anomalous dimension. The convenience of this classification is that webs can only mix (under gauge transformations) with other webs that connect the same number of lines. Thus, an independent basis of colour factors for $m$-line webs must be worked out for each value of $m$ separately.

Consider first diagrams which connect $n+1$ lines at $n$-loop order. The possible connected colour factors are shown for the case $n=3$ in figure 7 , where we use a notation in which the Wilson lines are separated from each other for ease of viewing each gluon diagram (in principle, these Wilson lines all originate from the same hard interaction point). In the connected colour factor, each Wilson line emits a single gluon only, as this is the only way in which the maximal number of lines can be connected. There are three such colour factors, only two of which are independent due to their being related by a Jacobi identity. The notation of figure 7 makes clear that one can view each connected colour factor as a 4-point scattering process, where the external gluons are attached to a Wilson line. Furthermore, each scattering process is at tree-level, which is a direct consequence of the fact that $n+1$ lines are connected at $n$-loop order: one can only form loops by removing a gluon from an external line, such that at most $n$ lines are connected. The number of independent colour factors at $n$-loop order then amounts to counting the number of independent colour factors in an $(n+1)$-point tree-level scattering amplitude. This is known to be $(n-1)$ ! (see e.g. ref. [96]). 

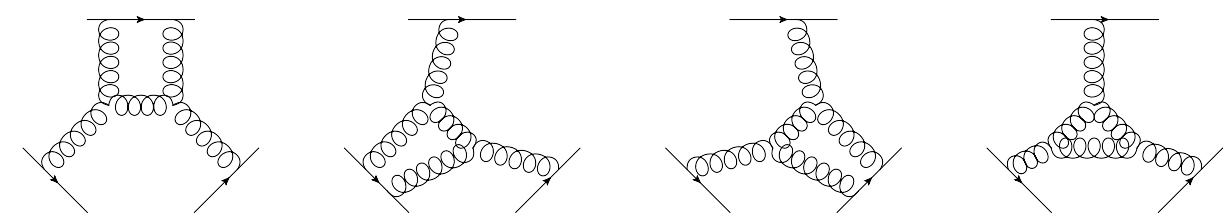

Figure 8. Possible connected colour factors connecting three Wilson lines at three-loop order.

For webs which link $m<(n+1)$ lines at $n$-loop order, the relevant connected colour factor graphs may have tree or loop-level topologies. That is, starting from the case of $n+1$ lines above, every time one disconnects a given external line from the diagram, one may either connect to another line more than once, or create a loop. For example, we show the case of three lines at three-loop order in figure 8. Four possible connected colour factors are shown, three of which are tree-level graphs, and one of which is loop-level (and indeed can be seen to be proportional to the two-loop $Y$ graph). ${ }^{3}$ For webs connecting progressively fewer lines at a given order, we can make the following general observation:

Webs which span $1 \leq m \leq(n+1)$ Wilson lines at $n$-loop order contribute colour factors corresponding to connected gluon graphs, whose topologies contain up to $n+1-m$ loops.

For a given value of $m$, the question arises of how many independent connected colour factors receive contributions from a given web (namely, the rank of the web-mixing matrix). It is possible to provide a simple formula for the case $m=n+1$, namely those webs which connect $n+1$ lines with $n$ single gluon emissons, such that there are no three or four-gluon vertices off the Wilson lines (one such example is the $(1,2,2,1)$ web of figure 5 ):

For webs in which $n+1$ external lines are connected by $n$ single gluon exchanges, the rank of the web-mixing matrix is given by

$$
r=\prod_{i=1}^{n+1}\left(n_{i}-1\right) !
$$

where $n_{i}$ is the number of gluon emissions on line $i$.

To show this result, one may use the effective vertex formalism for webs introduced in ref. [91], and which was used there to prove the fully connected colour factor property. As explained in detail in that paper, products of Wilson line operators may be rewritten to involve ordinary (rather than path-ordered) exponentials, thus providing a natural setting in which to use replica arguments (see refs. $[55,88]$ ) to prove the connected colour factor property. This introduces an infinite tower of effective vertices, where each " $k$-vertex" describes the emission of $k \geq 1$ gluons from a single Wilson line. Each such vertex consists of a superposition of $(k-1)$ ! independent fully connected colour factors, with associated kinematic coefficients. In this language, diagrams which contribute to the exponent of a

\footnotetext{
${ }^{3}$ Note that the 3 -line colour basis presented in ref. [91] consists of a superposition of the colour factors in figure 8.
} 


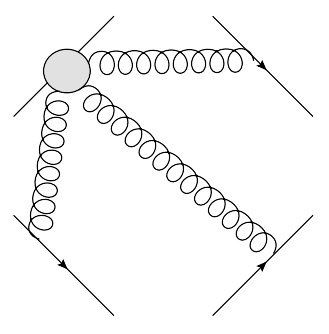

(a)

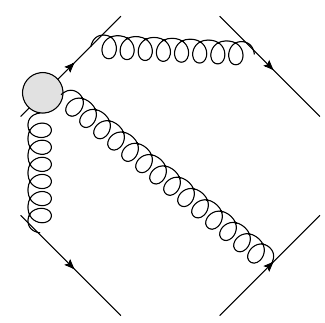

(b)

Figure 9. (a) Possible diagram for the (3,1,1,1) web, involving an effective 3-vertex on line 1; (b) Configuration involving a 2-vertex and a 1-vertex on line 1 .

Wilson line correlator are produced by linking $k$-vertices on each Wilson line such that the overall diagram has a single connected piece.

As we have remarked above, the class of webs we are considering gives rise to connected colour factors in which there is at most a single gluon emission from each of the $n+1$ external lines. This in turn means that there can be only one effective vertex on each line. As an example, consider the case of a $(3,1,1,1)$ web. In figure $9(\mathrm{a})$, we show one possible diagram involving a 3 -vertex on line 1 . This results in a fully connected configuration of gluons. Alternatively, one may consider a 2 -vertex and a 1 -vertex on line 1 - which gives a disconnected gluon diagram, as shown in figure $9(\mathrm{~b})$. Being disconnected, the latter does not contribute to the exponent.

Having established that for webs connecting $n+1$ lines with $n$ gluons each connected colour factor arises from diagrams in which the $i^{\text {th }}$ Wilson line contains a single $n_{i}$-vertex, the total number of independent connected colour factors arising from each web is simply the product of the number of independent connected colour factors in each $n_{i}$-vertex, which (from above) is $\left(n_{i}-1\right)$ !. This gives the result of eq. (3.1), as desired. We will see special cases of this result later in the paper, and also connect it to the results of ref. [95].

When fewer than $n+1$ lines are linked by gluon exchanges, it is no longer true that connected colour factor diagrams can only be formed by having single effective vertices on each line (plenty of examples can be found in ref. [91]). Nevertheless, it would be interesting to try to extend the above result, albeit after first solving for the total number of independent connected colour factors one expects for $m$-line webs with $m<(n+1)$. This appears to be a difficult combinatorial problem in general. Note that ref. [95] derived a result for the $(2,2, \ldots, 2)$ web, namely that the rank of the mixing matrix for $n$ gluon exchanges is given by $(n+1)$.

In this section, we have provided a simple formula for the rank of the mixing matrices for a special class of webs, namely those consisting of multiple gluon exchanges linking $n+1$ Wilson lines at $n$-loop order. In fact, for certain of these webs one may go much further, and explicitly solve for the entire web-mixing matrix. To this end, and motivated by the pure mathematical study of ref. [95], it is useful to relate web diagrams to partially ordered sets (posets), whose properties we review in the following section. 


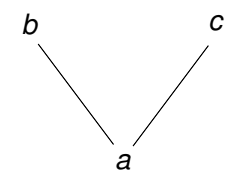

Figure 10. Example Hasse diagram for the poset $\{a, b, c\}$, with $a \leq b$ and $a \leq c$.

\section{Webs and posets}

In section 2 , we have reviewed what is known about web mixing matrices, with a particular emphasis on how they can be obtained combinatorially via overlap functions. In this section, we introduce mathematical ideas regarding partially ordered sets (posets). These were used in ref. [95] to study web mixing matrices, and will be reviewed here as a precursor for presenting the results of that paper. Furthermore, in what follows we will be able to use these poset ideas to obtain an explicit solution for the web-mixing matrix for $(1,2,2, \ldots, 2,1)$ webs, and investigate the weighted column sum rule in this context. Given that posets are not necessarily well-known to a physics audience, we will summarise relevant facts about them here in order to make our presentation self-contained. A pedagogical introduction may be found in e.g. ref. [97].

A poset is a set of objects $X$ endowed with an ordering operation $\leq$ possessing the following properties:

1. Reflexivity. If $x \in X$ then $x \leq x$.

2. Antisymmetry. If $x \leq y$ and $y \leq x$ then $x=y$.

3. Transitivity. If $x \leq y$ and $y \leq z, x \leq z$.

This ordering operation applies to pairs of elements in the set $X$, but such that not all pairs are necessarily ordered with respect to each other (hence the "partially" in "partially ordered set"). A familiar example of a poset is the set of integers, where $\leq$ has its usual meaning of "less than or equal to". In this case, however, the set is fully ordered, with each element being ordered with respect to every other element.

The structure of a given poset can be represented on a Hasse diagram. In such diagrams, each element of $X$ is represented by a symbol, and a line drawn between distinct elements $x$ and $y$ only if $x \leq y$ and $\{z \in X: x \leq z \leq y\}=\{x, y\}$. Then $x$ is drawn below $y$ to indicate the ordering. An example is shown in figure 10, for the set of elements $\{a, b, c\}$, such that $a \leq b, a \leq c$ but no ordering is implied between elements $b$ and $c$.

Returning to the web diagrams introduced in section 2, every web diagram $D$ can be identified with a poset. The elements of the poset are the various irreducible subdiagrams that occur in the diagram $D$. The ordering operation $\leq$ is defined as follows: given two irreducible subdiagrams $x$ and $y, x \leq y$ if $x$ lies closer to the origin than $y$, in the sense that one cannot pull $y$ into the origin without also pulling in $x$. As an example, consider the diagram shown in figure 11(a). In this diagram, the two gluons labelled $A$ form an irreducible subdiagram: the fact that they are crossed means that one cannot shrink either 


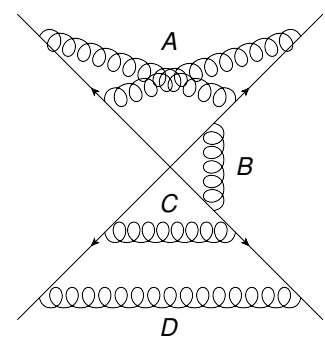

(a)

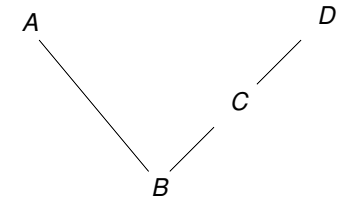

(b)

Figure 11. (a) Example web diagram; (b) Hasse diagram corresponding to the poset generated by the diagram in (a).

gluon to the origin independently of the other one. The single gluon exchanges labelled $B$, $C$ and $D$ are also irreducible subdiagrams. Then the structure of the diagram is encoded by the information that $B$ must be shrunk before $A, C$ or $D$, and $C$ must be shrunk before $D$. This corresponds to the Hasse diagram shown in figure 11(b).

Each diagram $D \in W$, for some web $W$, corresponds to a different Hasse diagram. One may then consider the set of Hasse diagrams instead of the original Feynman diagrams. Note that each Hasse diagram in a given web does not necessarily have the same number of vertices. This is because irreducible subgraphs from one web diagram may combine to make a higher-order irreducible piece in a different web diagram. An example is the crossed gluon pair in figure 11(a): there is another diagram in the set in which the two gluons are uncrossed, and thus appear as two separate vertices in the corresponding Hasse diagram. It should be clear from these remarks that all web diagrams can be represented in terms of posets, including those with three and four-gluon vertices off the Wilson lines. All of the diagrams in a given web have Hasse diagrams whose vertices are elements of the set of all possible irreducible subdiagrams.

The question of whether a given web diagram can be made out of partitions of another web diagram can in principle be rephrased in terms of the Hasse diagrams of the posets of the two diagrams (we will see examples of this later in the paper). However, note that partitions of a web diagram do not necessarily correspond to simple colourings of the vertices of its Hasse diagram. An example is again provided by the crossed gluon pair in figure 11(a). Partitions exist in which the two gluons are painted different colours. Multiplying together the elements of such a partition cannot produce a crossed gluon pair, and thus can only produce diagrams in which $A$ is replaced by two vertices, each representing a single gluon exchange.

For webs in which all irreducible subdiagrams are connected, there indeed exists a welldefined correspondence between colourings of the web diagrams $D \in W$, and colourings of the vertices of the corresponding Hasse diagrams. In this paper, we will be concerned with special cases of this, in which: (a) web diagrams consist exclusively of irreducible subdiagrams containing multiple gluon exchanges only; (b) any two Wilson lines have at most one gluon exchange between them. For the special cases we consider, the Hasse diagrams have a simple form, allowing a full solution of the relevant web-mixing matrices. 

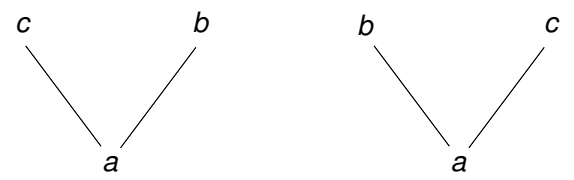

Figure 12. The two linear extensions of the poset shown in figure 10 .

As well as the above notions relating to posets, we will also need the concept of a linear extension in what follows. A linear extension of a given poset $P=(X, \leq)$ is defined as a permutation of the elements of $X$, such that all pairwise ordering relations remain satisfied. A simple example is provided by the poset whose Hasse diagram is shown in figure 10. This is specified by the set $\{a, b, c\}$ and the relations $a \leq b, a \leq c$. There are in fact two Hasse diagrams consistent with this ordering, shown in figure 12, and thus the number of linear extensions for this poset is equal to 2 . In general the classification of linear extensions is complicated - it is at least known that the problem of counting the linear extensions of a poset is $P$-complete [98]. Given a poset $P$, we denote the set of linear extensions of $P$ by $\mathcal{L}(P)$. It can then be proven quite generally that the diagonal elements of any web-mixing matrix are given by ref. [95]

$$
R_{D D}=\sum_{\pi \in \mathcal{L}(P)} \frac{(-1)^{\operatorname{des}(\pi)}}{p\left(\begin{array}{c}
p-1 \\
\operatorname{des}(\pi)
\end{array}\right)} .
$$

Here $\pi$ is a given linear extension of the poset corresponding to diagram $D$, and $p$ the number of members in the set (i.e. the number of vertices in the corresponding Hasse diagram). As stated above, $\pi$ corresponds to a permutation of the elements of the set, and one may label this by a permutation of $[12 \ldots p]$. For example, we may label the two Hasse diagrams in figure 12 (each corresponding to a linear extension of the same poset) by [123] and [132], where we have identified $(a, b, c)=(1,2,3)$. Given a permutation from the original poset (in this case [123]) to one of its linear extensions, we may count the number of descents in this permutation. That is, the number of consecutive numbers $i j$ in the permutation such that $j<i$. This is denoted by $\operatorname{des}(\pi)$ in eq. (4.1), and in the above example one has $\operatorname{des}[123]=0$ and $\operatorname{des}[132]=1$.

Note that the process of sequentially shrinking subdiagrams to the origin, discussed above from the combinatorial perspective, directly relates to the ultraviolet subdivergences present in these diagrams. This was observed already in refs. [88, 92] and analysed in detail in ref. [90]. We will return to this point in section 6 .

Having introduced various concepts relating to posets, and their relationship to web diagrams, we next consider the application of these ideas to specific web families. This is the subject of the following section.

\section{General solutions for web-mixing matrices}

In the previous section, we presented the notion of a partially ordered set, and explained how this concept relates to web diagrams. Methods from the theory of posets were used in 


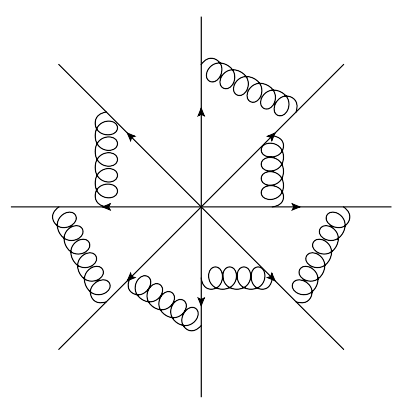

(a)

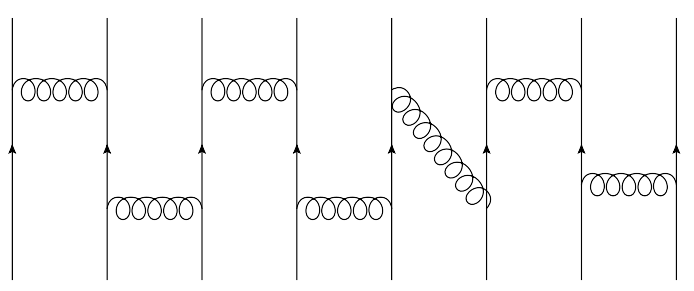

(b)

Figure 13. (a) An example $(1,2,2,2,2,2,2,1)$ web; (b) the same diagram "unwrapped", such that the hard interaction corresponds to a horizontal line at the bottom of the diagram.

ref. [95] to prove general properties of web-mixing matrices for two special cases, namely $(1,1, \ldots, 1, n)$ and $(1,2,2, \ldots, 2,1)$ webs, where we have used the notation introduced in section 2. An expression for the rank of mixing matrices for $(2,2, \ldots 2)$ webs was also given. Our aim here is not to reproduce the proofs contained in ref. [95], to which we refer the reader for all relevant details. Rather, our goal in the present study is to use the poset ideas from the previous section to go further than ref. [95] in solving for the web-mixing matrices of interest explicitly, to all orders in perturbation theory.

\subsection{The $(1,2,2, \ldots, 2,1)$ web}

In this section we consider webs in which at least one, and at most two attachments occur on any given leg. One may choose to label the external lines such that the pattern of exchanges is labelled by $(1,2, \ldots, 2,1)$. This notation does not fully specify the family of webs of interest: we will further assume that all consecutive lines are connected by gluon exchanges. An example is shown in figure 13(a), for the case of seven gluons connecting eight Wilson lines, and where all external lines are shown emanating from the same hard interaction vertex. It is also convenient to use an alternative representation of these diagrams, in which all external lines are shown parallel to each other. That is, one may redraw the diagram of figure 13(a) as shown in figure 13(b). Shrinking gluons to the origin in the former amounts to sinking gluons in the latter, and the hard interaction can be thought of as a horizontal line at the bottom of the diagram.

The $(1,2,2, \ldots 1)$ web family is of physical interest because it connects $n+1$ external lines with $n$ gluons. This is the maximum possible number of external lines which can be entangled at a given loop order, and corresponds to the class of webs whose rank was discussed in section 3. A given number of gluon exchanges $n$ specifies a unique $(1,2,2, \ldots 1)$ web (other similar webs at a given loop order can be obtained by permuting the external lines). The number of diagrams in this web is equal to the dimension of the permutation group which acts on the gluon emission vertices, namely

$$
\operatorname{dim}[S_{1} \times \underbrace{S_{2} \times S_{2} \ldots S_{2}}_{n-1 \text { factors }} \times S_{1}]=2^{n-1} .
$$



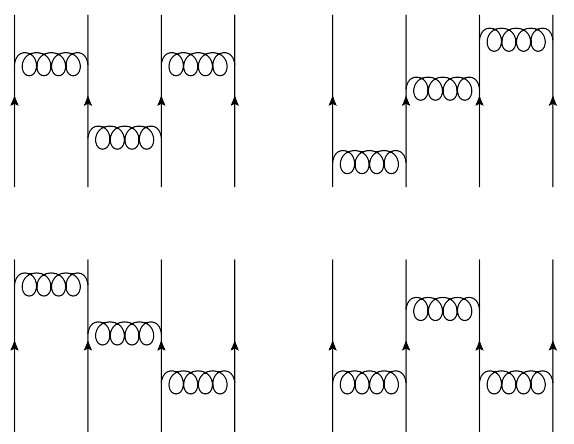

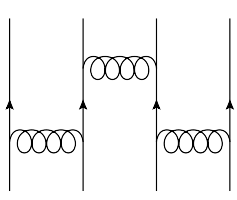

(a)

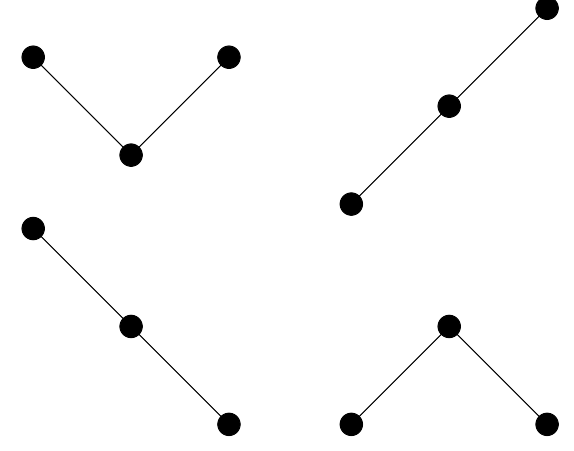

(b)

Figure 14. (a) The $(1,2,2,1)$ web redrawn as in figure 13(b); (b) corresponding Hasse diagrams.

We collect explicit examples from $n=2$ to $n=4$ in appendix A, together with the relevant mixing matrices.

As stated above, this family has been considered in ref. [95], where some general properties of the web-mixing matrices for all $n$ have been proven. Of relevance for the current study is that, for these webs, one has

$$
\operatorname{Tr}\left[R_{(1,2, \ldots, 2,1)}\right]=1 .
$$

Given the rank is equal to the trace for an idempotent matrix, this provides a special case of eq. (3.1), which dictates that the mixing matrix of any $(1,2,2, \ldots, 2,1)$ web has unit rank. Thus, there is only one linearly independent row or column. Here we use this property, together with the structure of the Hasse diagrams corresponding to each web diagram, to write down a solution for the web-mixing matrix for any $n$, extending the results of ref. [95].

The first thing to do is to relate the diagrams in a $(1,2,2, \ldots, 2,1)$ web to Hasse diagrams. We can do this by drawing the diagrams in the form shown in figure 13(b), and then associating a vertex $\bullet$ with each gluon exchange. This is possible because all irreducible subdiagrams correspond to a single gluon exchange. An example is shown in figure 14 for the $(1,2,2,1)$ web. Note that all the Hasse diagrams correspond to a single chain, which may or may not have kinks in. This is due to the special nature of the $(1,2, \ldots, 2,1)$ web, namely that each gluon exchange has only two neighbouring gluons (on adjacent external lines), and no other gluons exchanged between its own pair of external lines. This allows us to immediately write down the Hasse diagrams for any number of gluons $n$ : one simply draws all possible kink assignments in a chain with $n-1$ links. As a further example, the case of $n=4$ is shown in figure 15 .

The special form of these Hasse diagrams provides a basis for further study of the web-mixing matrix, and also the shrinking factors $s(D)$ which enter the weighted column sum rule. In order to investigate the former, we need to be able to evaluate the overlap functions introduced here in section 2. That is, the number of ways of forming a diagram in a $(1,2, \ldots, 2,1)$ web from a given partition of some other diagram in the same web. To this end, one may first see what partitions look like in terms of Hasse diagrams. As discussed 

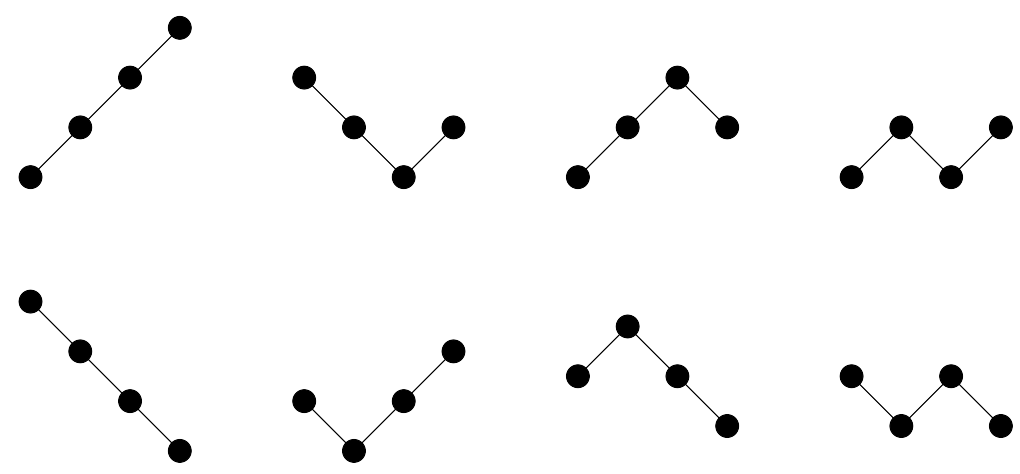

Figure 15. Hasse diagrams for the $(1,2,2,2,1)$ web.
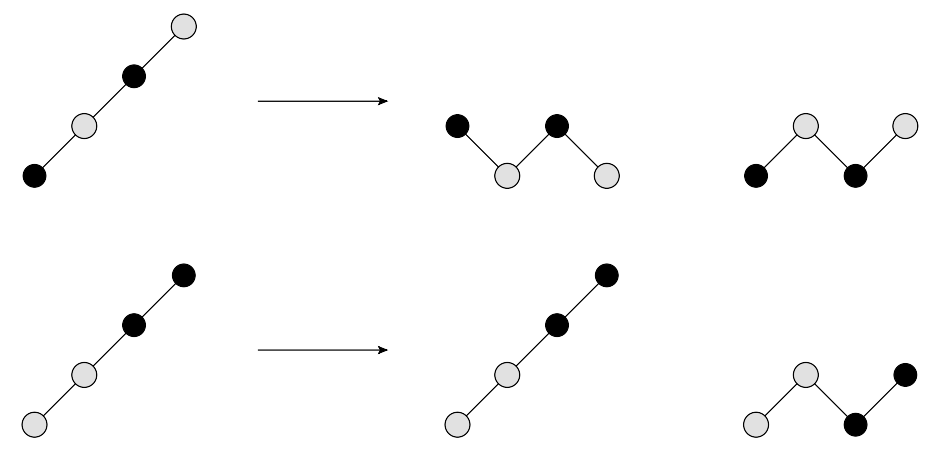

Figure 16. Example 2-partitions of a $(1,2,2,2,1)$ web diagram, and the diagrams formed by taking products of the partition elements.

in section 2, a partition corresponds to a colouring of the connected subdiagrams in a given web diagram. In the present case, this amounts to a colouring of gluons, namely of the vertices of the Hasse diagram. A given $m$-partition separates the Hasse diagram into $m$ groups of vertices. Furthermore, the multiplication rule for web diagrams easily translates into a multiplication rule for the elements of a partitioned Hasse diagram. Examples with $n=4$ are shown in figure 16, which shows two different 2-partitions of the same web diagram, and the diagrams obtained by taking products of the partition elements. One sees that the effect of the colouring is to break links between consecutive vertices if these have different colours; otherwise links are left intact. In each product, the grey element of the partition can be placed above or below the black element, due to the fact that the product of the related Feynman diagrams corresponds to placing subgraphs further or nearer to the hard interaction. It is straightforward to generalise this example to higher numbers of gluons $(n)$, and number of colours $(m)$.

We can now use these ideas to solve explicitly for a particular column of the webmixing matrix. We start by considering the Hasse diagram consisting of a chain with no kinks, such that each vertex is above the vertex to its left. For the $(1,2,2,1)$ case, this is the diagram in the upper-right of figure 14(b); for $n=4$, this is the first diagram in figure 15 . It is clear that such a diagram exists for any value of $n$, and we denote this diagram generically by $\uparrow$. It is then possible to find exactly the overlap functions $\left\langle\uparrow \mid P_{D}\right\rangle$, namely the 


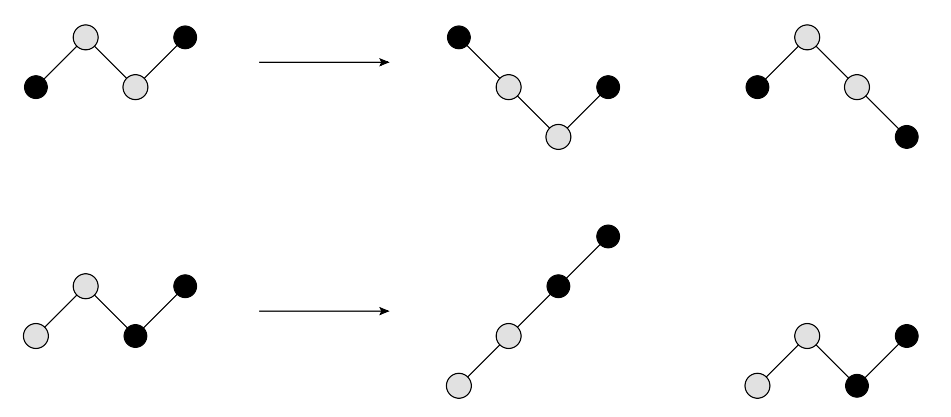

Figure 17. Example 2-partitions of the upper right-most diagram in figure 15, and the diagrams formed by taking products of the partition elements.

number of ways of making diagram $\uparrow$ from an arbitrary partition $P_{D}$ of diagram $D$ in the same web. One may then, in turn, solve for the values $R_{D \uparrow}$, namely the entire column of the web matrix corresponding to the diagram $\uparrow$.

The first step is to note that if $\uparrow$ can be made from the partition $P_{D}$, there is only one possible way to do this. This is because the various vertices of the Hasse diagram must be multiplied together in the unique sequence which generates the ascending pattern of $\uparrow$. Thus, in general the overlap function $\left\langle\uparrow \mid P_{D}\right\rangle$ can only be one or zero. Furthermore, it is clear that $\uparrow$ cannot be made out of partitions in which vertices of the same colour are separated by one or more vertices of a different colour. An example of this is shown in the upper diagram of figure 16, which has two grey vertices separated by a black vertex. In multiplying the partition elements together, the two grey vertices must occur at the same level, and either above or below the black vertices. This rules out the possibility of obtaining the diagram $\uparrow$, and this argument clearly generalises to higher numbers of gluons and colours.

It follows that $\left\langle\uparrow \mid P_{D}\right\rangle$ can only be non-zero for partitions in which groups of consecutive vertices are painted the same colour. Furthermore, in order to make $\uparrow$, none of these groups can contain a descending link. An example is shown in figure 17, in which we consider 2-partitions of the upper-rightmost diagram in figure 15. In the upper partition, a descending link is preserved by the colouring, which must necessarily survive in the diagrams formed by taking products of the partition elements. In the lower partition, however, the descending link is broken by choosing two different colours on either side of the link. The partition elements then consist solely of groups of ascending vertices, and it is then obvious that one can form $\uparrow$ from such a partition, as shown explicitly in figure 17.

Let us now consider a diagram $D$ whose Hasse diagram has $r$ descending links, and an $m$-partition of this diagram (equivalently, a colouring of $D$ with $m$ colours). In order to make $\uparrow$, the partition must be such that all descending links in the Hasse diagram are removed. This requires at least $r+1$ colours, such that we must have $m>r$ for $\left\langle\uparrow \mid P_{D}\right\rangle$ to be non-zero. After removing the descending links, any assignment of the remaining colours amongst the ascending links will give a partition which can reproduce $\uparrow$. Each of these assignments corresponds to a separate partition $P_{D}$, and the number of such partitions amounts to the number of ways of choosing $m-1-r$ links (the number of remaining colours) out of $n-1-r$ (the number of ascending links, which is the total number of links 
$(n-1)$ minus $r$ descending ones). Thus, one has

$$
\sum_{P_{D}, n\left(P_{D}\right)=m}\left\langle\uparrow \mid P_{D}\right\rangle=\Theta(m-r-1)\left(\begin{array}{c}
n-1-r \\
m-1-r
\end{array}\right),
$$

where $\Theta(n)$ is the Heaviside function, such that $\Theta(0)=1$. Substituting this result into eq. (2.3), one finds

$$
R_{D \uparrow}=\sum_{m=1}^{n} \frac{(-1)^{m-1}}{m} \Theta(m-r-1)\left(\begin{array}{c}
n-1-r \\
m-1-r
\end{array}\right)=\sum_{m=r+1}^{n} \frac{(-1)^{m-1}}{m}\left(\begin{array}{c}
n-1-r \\
m-1-r
\end{array}\right),
$$

where we have replaced the sum over individual partitions with a sum over the number of elements in a partition $m \equiv n\left(P_{D}\right)$. One may now set $p=n-1-r$ (the number of ascending links in the Hasse diagram for $D$ ) to get

$$
\begin{aligned}
R_{D \uparrow} & =\sum_{m=n-p}^{n} \frac{(-1)^{m-1}}{m}\left(\begin{array}{c}
p \\
m-n+p
\end{array}\right) \\
& =\sum_{s=0}^{p} \frac{(-1)^{n-s-1}}{n-s}\left(\begin{array}{l}
p \\
s
\end{array}\right),
\end{aligned}
$$

where we have set $s=n-m$ and used the symmetry property of binomial coefficients

$$
\left(\begin{array}{l}
p \\
s
\end{array}\right)=\left(\begin{array}{c}
p \\
p-s
\end{array}\right)
$$

We may now use the rather quirky identity (see e.g. eq. (5) of ref. [99])

$$
\left(\begin{array}{c}
n-1 \\
p
\end{array}\right)^{-1}=n \sum_{s=1}^{p}(-1)^{p-s}\left(\begin{array}{l}
p \\
s
\end{array}\right) \frac{1}{n-s}
$$

to get

$$
R_{D \uparrow}=\frac{(-1)^{n-p-1}}{n}\left(\begin{array}{c}
n-1 \\
p
\end{array}\right)^{-1}
$$

This is an important formula: it tells us the value of any element of the column of the webmixing matrix corresponding to diagram $\uparrow$, for any number of gluons! Furthermore, the only important quantity is seen to be the number of ascending links $p$ in the Hasse diagram of diagram $D$. Regarding the column as a whole, we can write a simple expression for the multiplicity $n_{p}$ of the element given by eq. (5.7). This will simply be the number of Hasse diagrams with $p$ ascending links. Given that there are $(n-1)$ links in each Hasse diagram in total, this corresponds to the number of ways of choosing $p$ links from $(n-1)$, namely

$$
n_{p}=\left(\begin{array}{c}
n-1 \\
p
\end{array}\right)
$$

A consistency check of this result is that the sum over all multiplicities is

$$
\sum_{p=0}^{n-1} n_{p}=\sum_{p=0}^{n-1}\left(\begin{array}{c}
n-1 \\
p
\end{array}\right)=2^{n-1}
$$


where we have used a well-known summation formula for binomial coefficients (see e.g. ref. [97]). The right-hand side is the dimension of the web-mixing matrix for the $(1,2, \ldots, 2,1)$ web with $n$ gluon exchanges, as it should be.

We can now go further than this. The fact that all columns are proportional to the column $R_{D \uparrow}$ (as follows from eq. (5.1)) means that we may write the elements of the mixing matrix as

$$
R_{D E}=R_{D \uparrow} a_{E},
$$

where $a_{E}$ are coefficients to be determined. A full solution for the web-mixing matrix now corresponds to finding the latter. To this end, we may use the result of ref. [91] namely, that left-eigenvectors of the web-mixing matrix are in one-to-one correspondence with connected colour factors. The $(1,2,2, \ldots, 2,1)$ web is special in that there is only one such connected colour factor [95], and it is straightforward to see how this arises diagramatically: the only way to form a fully connected colour factor is to form a commutator of the colour generators on each line which has two gluon emissions. ${ }^{4}$

One may show that the left-eigenvector $c_{D}$ with unit eigenvalue of the web-mixing matrix is given by:

$$
c_{D}=(-1)^{n-p_{D}-1},
$$

where $p_{D}$ is the number of ascending links in the Hasse diagram corresponding to $D$. To see this, one may use eqs. (5.7), (5.10) and (5.11) to find

$$
\sum_{D} c_{D} R_{D E}=\sum_{D} \frac{1}{n}\left(\begin{array}{c}
n-1 \\
p_{D}
\end{array}\right)^{-1} a_{E}
$$

We may convert the sum over diagrams $D$ into a sum over the number of ascending links $p$ in the corresponding Hasse diagrams, to get

$$
\sum_{D} c_{D} R_{D E}=\sum_{p=0}^{n-1} \frac{n_{p}}{n}\left(\begin{array}{c}
n-1 \\
p
\end{array}\right)^{-1} a_{E}=\sum_{p=0}^{n-1} \frac{1}{n} a_{E}=a_{E} .
$$

In the second step, we have used the result for the multiplicity factor $n_{p}$ from eq. (5.8). Equation (5.13) tells us that $c_{D}$ of eq. (5.11) is the left eigenvector we are looking for, provided

$$
c_{E}=a_{E}
$$

Another way to describe this left eigenvector is as follows:

The left eigenvector for the web-mixing matrix for a $(1,2, \ldots, 2,1)$ web is proportional to $c_{E}=(-1)^{t(E)}$, where $t(E)$ is the number of permutations of gluon emissions that takes diagram $\uparrow$ to diagram $E$.

This is consistent with the fact that the single connected colour factor for this web has a commutator of gluon generators on each external line that has two gluon emissions:

\footnotetext{
${ }^{4}$ In the effective vertex language of ref. [91], this amounts to having a 2-vertex on all lines which have two gluon emissions, in line with the comments in section 3 of the present paper.
} 
interchanging any pair of gluon emissions leads to a minus sign. An example is the $(1,2,2,1)$ case of figure 5 :

$$
\begin{aligned}
W^{(1,2,2,1)} & =\sum_{D, D^{\prime}} \mathcal{F}(D) R_{D D^{\prime}} C\left(D^{\prime}\right) \\
& =\underbrace{\frac{1}{6}(\mathcal{F}(a)-2 \mathcal{F}(b)-2 \mathcal{F}(c)+\mathcal{F}(d))}_{\mathcal{F}^{(1,2,2,1)}} \times \underbrace{(C(a)-C(b)-C(c)+C(d))}_{C^{(1,2,2,1)}}
\end{aligned}
$$

where

$$
\begin{aligned}
C^{(1,2,2,1)} & =T_{1}^{a}\left(T_{2}^{a b} T_{3}^{c b}-T_{2}^{b a} T_{3}^{c b}-T_{2}^{a b} T_{3}^{b c}+T_{2}^{b a} T_{3}^{b c}\right) T_{4}^{c} \\
& =-f^{a b c} f^{c b d} T_{1}^{a} T_{2}^{e} T_{3}^{d} T_{4}^{c}, \quad T_{i}^{a b} \equiv T_{i}^{a} T_{i}^{b} .
\end{aligned}
$$

Not only has the above analysis determined the left eigenvector of the web-mixing matrix with unit eigenvalue - it has also fixed the coefficients $a_{E}$ in eq. (5.10). We have thus explicitly obtained a general solution for the web-mixing matrix:

$$
R_{D E}=\frac{(-1)^{p_{D}+p_{E}}}{n}\left(\begin{array}{c}
n-1 \\
p_{D}
\end{array}\right)^{-1}
$$

where, as above, $p_{D}$ is the number of ascending links in the Hasse diagram of diagram $D$. We may check that this solution satisfies a number of constraints. Firstly, there is that of idempotence of the web-mixing matrix. The squared web-mixing matrix is given by

$$
\sum_{E} R_{D E} R_{E F}=\sum_{E}\left[R_{D \uparrow} c_{E}\right]\left[R_{E \uparrow} c_{F}\right]
$$

and we may simplify the right-hand side by observing

$$
\sum_{E} c_{E} R_{E \uparrow}=c_{\uparrow}=1 .
$$

Inserting this on the right-hand side of eq. (5.18) yields

$$
\sum_{E} R_{D E} R_{E F}=R_{D \uparrow} c_{F}=R_{D F}
$$

as required. The zero sum row rule is also satisfied, given that

$$
\sum_{D} c_{D}=0
$$

as follows from eq. (5.11). The reader may easily verify that the general solution reproduces the mixing matrices for the cases $n=2, n=3$ and $n=4$, which are collected in appendix A. For higher values of $n$, we may also compare eq. (5.17) with the general combinatorial formula for diagonal elements of web-mixing matrices, presented in eq. (4.1). A precise comparison at all orders requires a more involved understanding of linear extensions of the kinked chain than has been presented here (although we return to this point in section 6). Nevertheless, one may note that eq. (4.1) also involves the inverse binomial coefficients. 
One may also check that our solution satisfies the weighted column sum rule of eq. (2.6). However, this is not so straightforward given that one must first elucidate the structure of the $s(D)$ values for each web. We postpone a discussion of this point to section 6 .

As an example of the power of the above solution, we can present an explicit solution for the only surviving combination of kinematic factors that survives from the $(1,2,2, \ldots, 2,1)$ web. From eqs. (1.2) and (5.10), we see that the contribution of each web has the form

$$
\begin{aligned}
W^{(1,2,2, \ldots, 2,1)} & =\sum_{D E} \mathcal{F}_{D} R_{D E} C_{E} \\
& =\left(\mathcal{F}_{D} R_{D \uparrow}\right)\left(c_{E} C_{E}\right),
\end{aligned}
$$

where $\mathcal{F}_{D}$ and $C_{D}$ are the vectors of kinematic and colour parts. Using eq. (5.7) for $R_{D \uparrow}$, the kinematic combination is given by

$$
\sum_{D} R_{D \uparrow} \mathcal{F}_{D}, \quad R_{D \uparrow}=\frac{(-1)^{n-p_{D}-1}}{n}\left(\begin{array}{c}
n-1 \\
p_{D}
\end{array}\right)^{-1} .
$$

It is useful to clarify the above formula by collecting the first few non-trivial examples. Firstly there is the $n=2$ case, namely the web shown in figure 1 . The surviving kinematic combination is

$$
\mathcal{F}^{(1,2,1)}=\frac{1}{2}[\mathcal{F}(a)-\mathcal{F}(b)],
$$

using the labels from the figure. The $n=3$ case is shown in figure 5 , and using the labels from the figure one has

$$
\mathcal{F}^{(1,2,2,1)}=\frac{1}{6}[\mathcal{F}(a)-2 \mathcal{F}(b)-2 \mathcal{F}(c)+\mathcal{F}(d)] .
$$

The $n=4$ case is shown in figure 20, and contributes the kinematic combination

$$
\begin{aligned}
\mathcal{F}^{(1,2,2,2,1)}=\frac{1}{24} & {[6(\mathcal{F}[[1],[2,1],[3,2],[4,3],[4]]-\mathcal{F}[[1],[1,2],[2,3],[3,4],[4]])} \\
& -2(\mathcal{F}[[1],[1,2],[2,3],[4,3],[4]]+\mathcal{F}[[1],[2,1],[2,3],[3,4],[4]] \\
& +\mathcal{F}[[1],[1,2],[3,2],[3,4],[4]]-\mathcal{F}[[1],[1,2],[3,2],[4,3],[4]] \\
& -\mathcal{F}[[1],[2,1],[3,2],[3,4],[4]]-\mathcal{F}[[1],[2,1],[2,3],[4,3],[4]])] .
\end{aligned}
$$

As will be detailed elsewhere [93], it is possible to develop systematic methods for carrying out the integration of the kinematic factors for a general number of gluon exchanges. Combined with the above results, this would provide a complete solution of this web family to all orders in perturbation theory, and strongly motivates the investigation of more general webs.

\subsection{The $(1,1,1, \ldots, 1, n)$ web}

In the previous section, we have discussed the $(1,2,2, \ldots, 2,1)$ web at length, and given a full solution for the web-mixing matrix for an arbitrary number of gluon exchanges. In this section, we present a solution for a different web family, namely that of $(1,1,1, \ldots, n)$. 


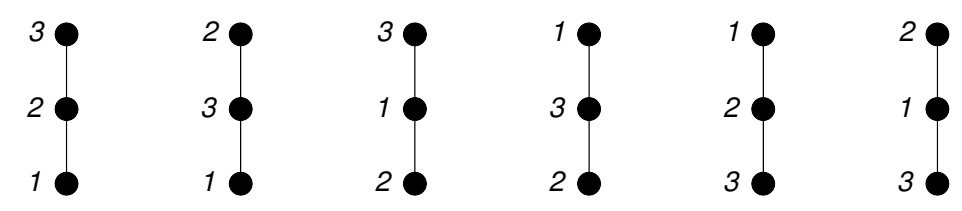

Figure 18. Hasse diagrams for the $(1,1,1,3)$ web.

This is in fact a shorter exercise than the previous case, as most of the necessary results have already been derived in ref. [95].

The Hasse diagrams for the $(1,1, \ldots, 1, n)$ case consist of $n$ ! vertical chains, each corresponding to a permutation of the gluon emissions on the line which has $n$ gluon emissions. An example is shown, for the $n=3$ case, in figure 18. Consequently, one can label each diagram by a permutation $\pi$ of $[12 \ldots n]$. A general element of the web-mixing matrix can then be denoted $R_{D D^{\prime}} \equiv R_{\pi \sigma}$, where $\pi$ and $\sigma$ are the permutations corresponding to diagrams $D$ and $D^{\prime}$ respectively. We then have the following result: ${ }^{5}$

The web-mixing matrix element $R_{\pi \sigma}$ is given by

$$
R_{\pi \sigma}=\frac{(-1)^{n-r_{\pi \sigma}-1}}{n}\left(\begin{array}{c}
n-1 \\
r_{\pi \sigma}
\end{array}\right)^{-1}
$$

where $r_{\pi \sigma}$ is the number of ascents in the permutation that takes $\sigma$ to $\pi$.

The definition of the latter quantity, and how to find the web-mixing matrix element, is best clarified by a simple example. To this end, we consider the web of figure 21, whose web-mixing matrix is shown in eq. (A.4). The ordering of diagrams shown in the figure can be labelled by the permutations 123, 132, 312, 321, 213 and 231, where these denote the ordering of the gluons (moving outwards from the hard interaction) on the Wilson line which has three gluon emissions. Consider now the $(3,2)$ element of the matrix, corresponding to $R_{(312),(132)}$. The permutation that takes 312 to 132 has 1 ascent (in other words, one consecutive pair of increasing numbers; to see this, note that it is the same permutation as that which takes 123 to 213), and thus from eq. (5.27) one expects

$$
R_{(123),(132)}=\frac{(-1)^{3-1-1}}{3}\left(\begin{array}{c}
3-1 \\
1
\end{array}\right)^{-1}=-\frac{1}{6}
$$

as indeed is observed. As a second example, consider the $(2,6)$ element of the matrix, corresponding to $R_{(132,231)}$. The permutation which takes 132 to 231 would take 123 to 321 , and thus has 0 ascents. Thus one expects

$$
R_{(123),(132)}=\frac{(-1)^{3-1-0}}{3}\left(\begin{array}{c}
3-1 \\
0
\end{array}\right)^{-1}=\frac{1}{3}
$$

as observed.

\footnotetext{
${ }^{5}$ Note that we have rephrased the definition of $r_{\pi \sigma}$ from ref. [95], where it was given in terms of descents rather than ascents. One is allowed to do this due to the reflection property of binomial coefficients.
} 
Some comments are in order. First, the web-mixing matrices for this family are symmetric i.e. $R_{\pi \sigma}=R_{\sigma \pi}$. This follows from the definition of $r_{\pi \sigma}$, and the fact that the number of ascents in the permutation $\sigma \rightarrow \pi$ is the same as the number of descents in the inverse permutation $\pi \rightarrow \sigma$. That is,

$$
r_{\pi \sigma}=(n-1)-r_{\sigma \pi}
$$

Swapping $r_{\pi \sigma} \rightarrow r_{\sigma \pi}$ thus leaves the right-hand side of eq. (5.27) invariant, via the reflection property of binomal coefficients. Secondly, there is a remarkable similarity between the expression of eq. (5.27), and the formula for the possible values in the $(1,2,2, \ldots, 1)$ case, eq. (5.17). This is because the Hasse diagrams in the present case are governed by permutations of $[12 \ldots n]$, where these permutations can be classified by the number of ascents and descents, and thus mapped to kinked chain diagrams. The multiplicities of the various values in this case, however, are different, as we discuss below.

Equation (5.27) has a number of corollaries. Firstly, the permutation that takes a diagram to itself has $n-1$ ascents (i.e. it takes $[12 \ldots n]$ to itself). Thus, the diagonal elements are given by

$$
R_{\pi \pi}=\frac{1}{n}
$$

This can clearly be observed in the examples in appendix A. It is also possible to derive this result directly from eq. (4.1), which gives a general formula for the diagonal elements of any web-mixing matrix (in our case, the number of vertices in the Hasse diagram is $p=n$ ). The poset for each web diagram has a fully ordered Hasse diagram (e.g. figure 18), thus has only one linear extension. Furthermore, the number of descents in the permutation which takes a diagram to itself is zero, and thus eq. (5.29) must correspond to eq. (4.1) with $\operatorname{des}(\pi)=0$. This is indeed the case.

Knowledge of the diagonal elements allows us to obtain a simple solution for the trace:

$$
\operatorname{Tr}\left[R_{(1,1, \ldots, 1, n)}\right]=(n-1) !,
$$

which follows from the fact that each diagonal element is $1 / n$, and there are $n$ ! of them. Again the trace corresponds to the rank, and thus one derives a special case of the general result of eq. (3.1).

Having solved for the possible elements of the web-mixing matrix, it is also convenient to note their multiplicities in each row and column. These are given by the following result [95]:

Identifying $r \equiv r_{\pi \sigma}$, the values in a given row or column of the web-mixing matrix are given by eq. (5.27) with all possible values of $r$. The multiplicity of each value is given by the Eulerian number $\left\langle\begin{array}{l}n \\ r\end{array}\right\rangle$.

Given that the Eulerian numbers may not be generally familiar to the reader, we briefly introduce their properties in appendix B. As clarification of their role as multiplicities in web-mixing matrices, consider the $n=4$ case, whose web-mixing matrix is shown in eq. (A.5). In any given row or column, the possible values are given by eq. (5.27) 
with $r=0,1,2,3$, which gives $-1 / 4,1 / 12,-1 / 12$ and $1 / 4$ respectively. Furthermore, these should have multiplicities 1, 11, 11 and 1, as given by the relevant Eulerian numbers from table 2. This is indeed observed.

Note that the sum of multiplicities in any given row or column is

$$
\sum_{r=0}^{n-1}\left\langle\begin{array}{l}
n \\
r
\end{array}\right\rangle=n !
$$

where we have used a known identity of Eulerian numbers (in words: summing over $r$ the number of permutations with $r$ ascents must give the total number of permutations, which is $n$ !). The right-hand side of eq. (5.31) is the dimension of the web-mixing matrix, as it should be. Note that the sum over elements in any given row is

$$
\sum_{r=0}^{n-1} \frac{(-1)^{n-r-1}}{n}\left(\begin{array}{c}
n-1 \\
r
\end{array}\right)^{-1}\left\langle\begin{array}{l}
n \\
r
\end{array}\right\rangle=0
$$

where we have used eq. (B.1). This is an explicit realisation of the zero sum row rule of eq. (2.5), which has been proven to be true fully generally in ref. [89]. A similar sum also ensures that for the $(1,1, \ldots, 1, n)$ web family, the weighted column sum rule is satisfied. We return to this point in section 6 .

In this section, we have presented solutions for two specific families of web-mixing matrices, for any number of gluon exchanges, extending the results of ref. [95]. Next, we consider the weighted column sum rule of ref. [90] in the poset language.

\section{The weighted column sum rule}

In this section, we return to a specific combinatorial property of web matrices, namely the weighted column sum rule of eq. (2.6). This was first conjectured in ref. [90], and is crucially related to the renormalisation of the hard interaction vertex at which the external eikonal lines meet. Based on this relation, it was shown in ref. [90] that for webs which consist solely of multiple gluon exchanges, the sum rule must hold. However, the sum rule appears to be more general than this, and was indeed satisfied in all examples considered in ref. [90], including those webs which contain multiple gluon vertices off the eikonal lines. This perhaps is not surprising, given the results of ref. [91], which show that webs containing multiple gluon exchanges are intimately linked with webs which have multiple gluon vertices, by virtue of sharing the same connected colour factors. They are also linked by gauge transformations.

Given the above remarks, it is useful to study the weighted column sum rule from a purely combinatorial point of view. Whilst a full combinatorial proof for arbitrary webs remains elusive, we will relate some key concepts which arise from the poset language of section 4 , and also examine the sum rule for the particular cases of $(1,2, \ldots, 2,1)$ and $(1,1, \ldots, n)$ webs. 


\section{1 $s(D)$ and linear extensions}

Our first step is to tighten up the combinatorial definition of the number of ways of shrinking irreducible subdiagrams to the origin in a given web diagram. This may be stated as follows, for all classes of web:

Let $P$ be the poset corresponding to a given web diagram $D$. Then the number of ways of shrinking all irreducible subdiagrams to the origin is the number of linear extensions of $P$.

This follows from the definition of a linear extension which, as discussed in section 4, corresponds to a permutation of the elements of a poset which preserves the ordering requirements. The Hasse diagram for a web diagram $D$ containing $m$ irreducible subdiagrams contains $m$ vertices, one for each subdiagram. Each possible shrinking sequence must preserve the ordering of these vertices, and corresponds to a permutation of the vertices of the Hasse diagram. Thus, each shrinking sequence can be uniquely identified with a linear extension of the poset of $D$, such that the total number of shrinking sequences is equal to the number of linear extensions. As an example, consider the diagram of figure 11(a), whose Hasse diagram is shown in figure 11(b). There are three possible shrinking sequences, which we may denote by $B A C D, B C A D, B C D A$. This is indeed the number of linear extensions of the poset.

Now we recall the definition of $s(D)$ in ref. [90]. For diagrams where one can sequentially shrink each connected subdiagram independently to the vertex (i.e. which are maximally reducible), $s(D)$ is defined to be the number of shrinking sequences. For non-maximally-reducible diagrams, $s(D)=0$. It thus follows from the above discussion that for any maximally-reducible diagram, $s(D)$ can be identified with the number of linear extensions of the poset of $D$.

Having identified $s(D)$ in poset language, we may examine the weighted column sum rule for the particular cases of $(1,1, \ldots, n)$ and $(1,2,2, \ldots, 2,1)$ webs considered above. In both of these cases, all diagrams are maximally reducible, in that all irreducible diagrams consist of single gluon exchanges. Thus, $s(D)$ for each web diagram $D$ in either family can be simply identified with the number of linear extensions of the poset of $D$.

\subsection{Column sum rule for $(1,1, \ldots, 1, n)$ webs}

For $(1,1, \ldots, 1, n)$ webs, an explicit solution for the web-mixing matrix elements has been given in eq. (5.27), where $r$ takes values $0, \ldots, n-1$, and the multiplicity of each value in a given column is given by the Eulerian number $\left\langle\begin{array}{l}n \\ r\end{array}\right\rangle$. The $s(D)$ values for any web in this family are trivial, in that they always have the form

$$
s(D)=\left(\begin{array}{lll}
1 & 1 & 1
\end{array} \ldots 1\right)
$$

This is easily seen from the fact that the Hasse diagram for any $(1,1, \ldots, 1, n)$ web diagram is fully ordered (e.g. figure 18), and thus all gluon exchanges can only be shrunk in a single sequence, beginning with the gluon closest to the hard interaction, and working outwards. 
Contracting the vector of $s(D)$ values with any column of the web-mixing matrix then gives

$$
\begin{aligned}
\sum_{D} s(D) R_{E D} & =\sum_{r=0}^{n-1} \frac{(-1)^{n-r-1}}{n}\left(\begin{array}{c}
n-1 \\
r
\end{array}\right)^{-1}\left\langle\begin{array}{l}
n \\
r
\end{array}\right\rangle, \quad \forall E \\
& =0
\end{aligned}
$$

where we have used eq. (B.1). Note that this is analagous to the proof of the zero sum row property for these webs, as performed in eq. (5.32). This is due to the fact that the same values occur with the same multiplicities in any given row or column of the web-mixing matrix, and also crucially depends on the fact that $s(D)$ is the same for all diagrams.

The fact that the row and column rules appear to be related for this web family (a consequence in this special case of symmetry of the web-mixing matrix) is interesting by itself. The row rule is known to be related to colour properties of web diagrams, including the $1 / N_{c}$ expansion [89]. On the other hand, the column rule is related to kinematic information, namely the structure of singularities [90]. A link between the row and column rules is then a form of colour-kinematic duality, which may be related to other such dualities in the literature. It would be interesting to investigate the possible relationship between the row and column rules in more detail and for more general webs. Another possible question is that of whether there is a sub-column rule analagous the sub-row rule introduced in ref. [90] via an expansion about the planar limit.

Here we have seen explicitly that the weighted column sum rule is satisfied, for this particular family, at the level of individual webs. Furthermore, this follows purely from combinatorial considerations. This is consistent with the fact that webs renormalise individually, with no mixing between webs. Next, we consider the $(1,2, \ldots, 2,1)$ case.

\subsection{Column sum rule for $(1,2, \ldots, 2,1)$ webs}

This web family makes an interesting counterpoint to that of the previous section. It is nontrivial in that the $s(D)$ values for each web are different for different diagrams $D$. This in turn implies that the weighted column sum rule is different to the zero sum row rule.

An explicit solution for a single column of the web-mixing matrix for the $(1,2, \ldots, 2,1)$ family (corresponding to diagram $\uparrow$ ) has been given in eq. (5.7), where each value occurs with a multiplicity given by the binomial coefficient of eq. (5.8). All other columns are proportional to this column as a consequence of eq. (5.1) (which together with idempotence implies that all web-mixing matrices for this family have unit rank). Hence, if the column sum rule is satisfied for the column $R_{E \uparrow}$, it is satisfied for all columns.

As stated in section $6.1, s(D)$ for a maximally reducible web diagram is the number of linear extensions of the poset corresponding to diagram $D$. In the present case, this corresponds to linear extensions of posets whose Hasse diagrams correspond to kinked chains (see e.g. figure 15). We collect the corresponding $s(D)$ values up to nine loop order, together with their multiplicities, in appendix C. As we describe in the appendix, a closed form solution for these values is not known. However, one may check the weighted column sum rule in particular cases, for the column $R_{D \uparrow}$. One must first identify which $s(D)$ values correspond to which diagrams. The value of $R_{D \uparrow}$ for each diagram is then given by 


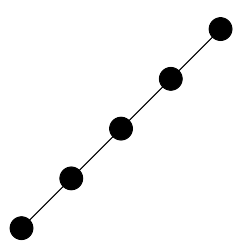

(a)

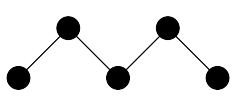

(e)

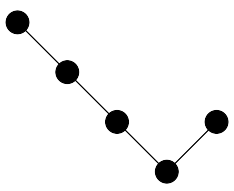

(i)

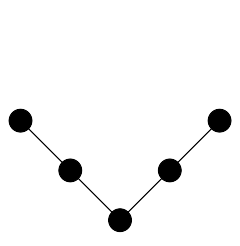

(m)

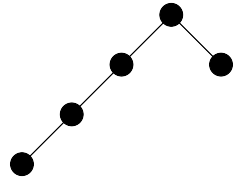

(b)

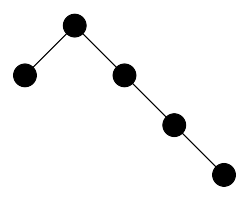

(f)

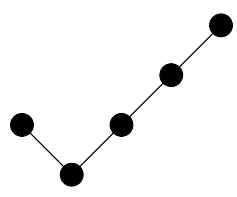

(j)

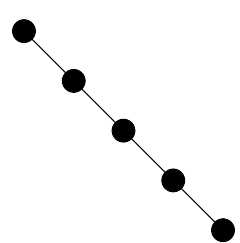

(n)

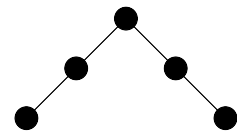

(c)

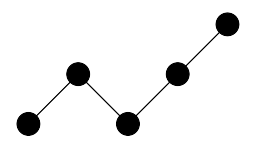

(g)

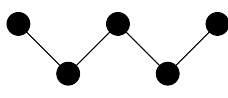

(k)

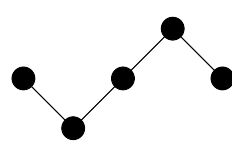

(o)

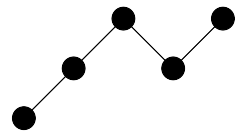

(d)

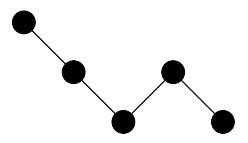

(h)

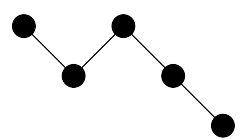

(I)

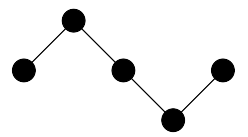

(p)

Figure 19. Hasse diagrams for the $(1,2,2,2,2,1)$ web.

eq. (5.7). As an example, the $s(D)$ values for the $n=3$ case and corresponding to the diagrams in figure 14 are 2, 1,1 and 2 . The corresponding elements of $R_{D \uparrow}$ are $-1 / 6,+1 / 3$, $+1 / 3$ and $-1 / 6$. One then finds $\sum_{D} s(D) R_{D \uparrow}=0$ as required.

As a highly non-trivial example, one may consider also the case $n=5$. The Hasse diagrams corresponding to the 16 web diagrams are shown in figure 19, and the corresponding $s(D)$ factors and $R_{D \uparrow}$ values in table 1 . Note that we have multiplied the web-mixing matrices by $5 !=120$ in order to make the numbers easier to read. Contracting the $s(D)$ values with the web-mixing matrix elements gives

$$
\begin{aligned}
\sum_{D} s(D) R_{D \uparrow} \propto & {[1 \times 24 \times 2]+[4 \times(-6) \times 4]+[6 \times 4 \times 2] } \\
& +[9 \times(-6) \times 4]+[16 \times 4 \times 2]+[11 \times 4 \times 2] \\
= & 0,
\end{aligned}
$$

as expected if the weighted column sum rule is true. One sees from this particular example that the nature of the cancellations involved is highly non-trivial, involving an intimate interplay between the numbers of eq. (5.7), and the linear extensions of the kinked chain. 


\begin{tabular}{|c|c|c|c|c|c|}
\hline$D$ & $s(D)$ & $5 ! R_{D \uparrow}$ & $D$ & $s(D)$ & $5 ! R_{D \uparrow}$ \\
\hline$(\mathrm{a})$ & 1 & 24 & $(\mathrm{i})$ & 4 & -6 \\
$(\mathrm{~b})$ & 4 & -6 & $(\mathrm{j})$ & 4 & -6 \\
$(\mathrm{c})$ & 6 & 4 & $(\mathrm{k})$ & 16 & 4 \\
$(\mathrm{~d})$ & 9 & -6 & $(\mathrm{l})$ & 9 & -6 \\
$(\mathrm{e})$ & 16 & 4 & $(\mathrm{~m})$ & 6 & 4 \\
$(\mathrm{f})$ & 4 & -6 & $(\mathrm{n})$ & 1 & 24 \\
$(\mathrm{~g})$ & 9 & -6 & $(\mathrm{o})$ & 11 & 4 \\
$(\mathrm{~h})$ & 9 & -6 & $(\mathrm{p})$ & 11 & 4 \\
\hline
\end{tabular}

Table 1. The factors $s(D)$ for the diagrams of figure 19, together with the corresponding entries of the web-mixing matrix $R_{D \uparrow}$, where have multiplied by $5 !=120$.

A dramatic simplification occurs for even values of $n$. Consider a diagram $D$ and its conjugate diagram $D^{*}$ whose Hasse diagram is obtained from that of $D$ by reflection about the horizontal. The shrinking factors for these two diagrams are related by

$$
s(D)=s\left(D^{*}\right)
$$

which follows from the fact that the shrinking sequences for diagram $D^{*}$ can be obtained by reversing those for $D$. The Hasse diagram of $D^{*}$ will have $n-1-p$ ascending links if that of $D$ has $p$ ascending links, and thus from eq. (5.11) one may write

$$
R_{D \uparrow}=R_{D^{*} \downarrow}=(-1)^{n-1} R_{D^{* \uparrow}} .
$$

Hence, for even $n$ values, conjugate diagrams (which have the same $s(D)$ values) have column entries in the web-mixing matrix which are equal in magnitude but opposite in sign. The weighted column sum rule is then automatically satisfied, and thus proven for the even $(1,2,2, \ldots, 2,1)$ webs.

In this section, we have expressed the factors $s(D)$ occuring in the weighted column sum rule of eq. (2.6) in poset language, namely as the number of linear extensions of the poset corresponding to web diagram $D$, where this is maximally reducible. In the $(1,1, \ldots, 1, n)$ case, we see that the sum rule can indeed be proven combinatorially. The $(1,2,2, \ldots, 2,1)$ case is more intricate, and involves combinatorial quantities (linear extensions of kinked chain posets) about which little is currently known. However, we have proven that the sum rule is satisfied for individual webs, for an even number of gluon exchanges. Whilst a formal proof of the weighted column sum rule (also including other web families) remains elusive, we hope that the ideas of this section will be useful in the further study of this property.

\section{Conclusion}

The study of infrared singularities in non-Abelian gauge theories continues to be of interest, not least as this is intimately linked to the improvement of collider physics predictions through resummation of large logarithms. In this paper we have continued the development 
of a diagrammatic ("web") approach to soft gluon exponentiation or, equivalently, correlators of multiple Wilson lines. The web approach to multiparton scattering identifies closed sets of diagrams, whose kinematic and colour parts mix according to web-mixing matrices. This systematic organisation greatly simplifies the calculation of multiloop contributions, including the structure of subdivergences. A full understanding of the exponent necessitates the development of methods for carrying out the necessary kinematic integrals - and these are underway $[93,94]$ - but also the classification of the mixing matrices themselves. Here we have focused on the latter. Their pure combinatorial origin, already identified in ref. [89], has allowed us to relate the study of web-mixing matrices to that of partially ordered sets, a connection which was already explored in a pure mathematical context in ref. [95].

For a special class of webs, namely those consisting of $n+1$ Wilson lines linked by $n$ gluon exchanges, we have presented a simple formula for the rank of the mixing matrix. This tells us how many independent colour factors receive contributions from a given web, and it is an interesting combinatorial problem in its own right to extend this to more general webs.

Using the poset language of ref. [95], we have gone much further than studying the rank in being able to provide explicit solutions for two particular web families, namely the $(1,2,2, \ldots, 2,1)$ and $(1,1, \ldots, 1, n)$ webs. It is interesting to note that these are extremal cases of the special class mentioned above - they contribute 1 and $(n-1)$ ! independent colour factors respectively, where the total number of colour factors at a given order is known to be $(n-1)$ !. The formulae for the mixing matrices involve intriguing combinations of inverse binomial coefficients, matching the similar behaviour observed for diagonal elements of more general webs in ref. [95]. Combined with general methods for carrying out kinematic integrals, details of which will be presented elsewhere [93, 94], these results are potentially very powerful, allowing unprecedented insights into the all-order structure of the exponents of amplitudes. This provides strong motivation for generalising the results presented here, particularly to webs involving three and four-gluon vertices off the Wilson lines. However, it is also worth stressing that there may be gauges [24] in which the effect of some of these graphs can be absorbed in webs consisting of multiple gluon exchanges only, making the web-mixing matrices for the latter more physically relevant.

The poset language also allows some understanding of the weighted column sum rule for web-mixing matrices presented in ref. [90], which implements the cancellation of subdivergences. While a general combinatorial proof of this property remains elusive, the poset language seems to provide the right tools for such a proof. A deeper understanding of the columns of web-mixing matrices may provide insights into a perceived duality between subleading colour corrections, and subleading kinematic divergences, given the zero sub-row sum rule observed in ref. [89].

Further use of the poset langauge presented here and in ref. [95] (and possibly other combinatorial methods) can be used to generalise the results to other web families. Our ultimate aim is to probe the all-order structure of infrared singularities and the associated physical consequences, by elucidating where possible the structure of web-mixing matrices on the one hand, and kinematic integrals on the other. Progress on both of these fronts is ongoing. 


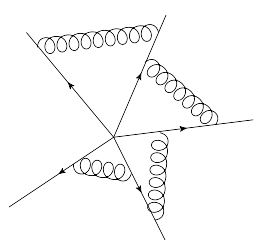

$[[1],[2,1],[3,2],[4,3],[4]]$

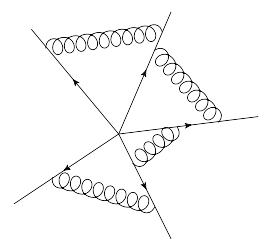

$[[1],[2,1],[3,2],[3,4],[4]]$

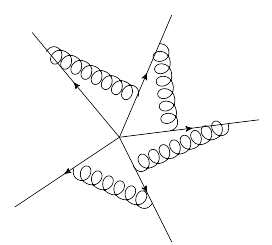

$[[1],[1,2],[2,3],[3,4],[4]]$

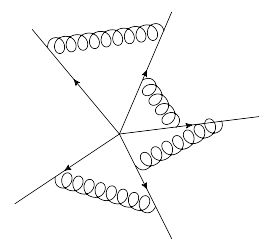

$[[1],[2,1],[2,3],[3,4],[4]]$

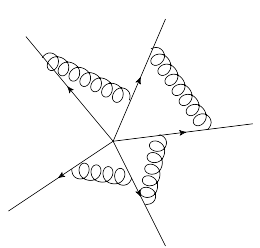

$[[1],[1,2],[3,2],[4,3],[4]]$

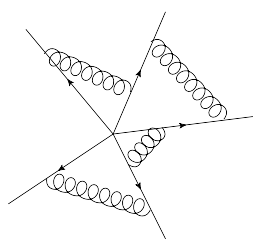

$[[1],[1,2],[3,2],[3,4],[4]]$

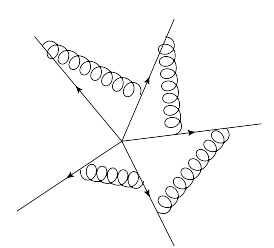

$[[1],[1,2],[2,3],[4,3],[4]]$

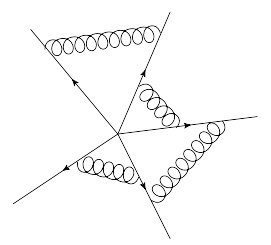

$[[1],[2,1],[2,3],[4,3],[4]]$

Figure 20. Web whose mixing matrix is given by eq. (A.3).

\section{Acknowledgments}

We thank Einar Steingrímsson and Jenni Smillie for discussions. CDW thanks the Higgs Centre for Theoretical Physics for repeated hospitality. CDW and EG are supported by the U.K. Science and Technology Facilities Council (STFC).

\section{A Examples of web-mixing matrices}

In this appendix, we collect explicit examples of web-mixing matrices for the $(1,2,2, \ldots 2,1)$ and $(1,1, \ldots, 1, n)$ families, where $n$ is the number of gluon exchanges in each case.

\section{A.1 $(1,2,2, \ldots 2,1)$ webs}

The web diagrams corresponding to the $n=2$ and $n=3$ cases are shown in figures 1 and 5 respectively. The corresponding web mixing matrices are:

$$
R_{(1,2,1)}=\frac{1}{2}\left(\begin{array}{rr}
1 & -1 \\
-1 & 1
\end{array}\right)
$$

and

$$
R_{(1,2,2,1)}=\frac{1}{6}\left(\begin{array}{rrrr}
1 & -1 & -1 & 1 \\
-2 & 2 & 2 & -2 \\
-2 & 2 & 2 & -2 \\
1 & -1 & -1 & 1
\end{array}\right)
$$

The $(1,2,2,2,1)$ web is shown in figure 20 , where we label each diagram according to a notation first introduced in ref. [88], and in which gluon exchanges associated with the same connected subdiagram are labelled by integers $i$. The $m$-tuple $\left[n_{1}, n_{2} \ldots, n_{m}\right]$ associated with each external line then labels the gluon emissions on that line, working inwards towards the hard interaction vertex. In this case the mixing matrix is 


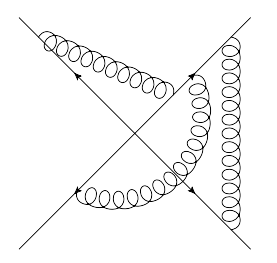

$[[1],[1,2,3],[3],[2]]$

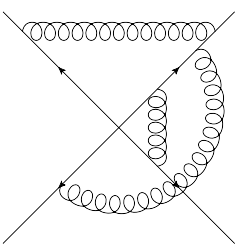

$[[1],[3,2,1],[3],[2]]$

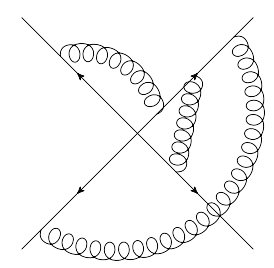

$[[1],[1,3,2],[3],[2]]$

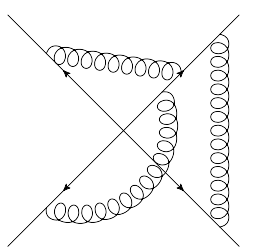

$[[1],[2,1,3],[3],[2]]$

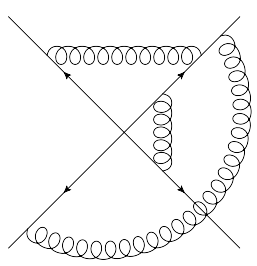

[[1],[3,1,2],[3],[2]]

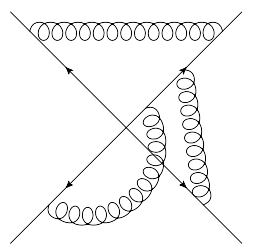

$[[1],[2,3,1],[3],[2]]$

Figure 21. Web whose mixing matrix is given by eq. (A.4).

$$
R_{(1,2,2,2,1)}=\frac{1}{24}\left[\begin{array}{rrrrrrrr}
6 & -6 & -6 & 6 & -6 & 6 & 6 & -6 \\
-6 & 6 & 6 & -6 & 6 & -6 & -6 & 6 \\
-2 & 2 & 2 & -2 & 2 & -2 & -2 & 2 \\
2 & -2 & -2 & 2 & -2 & 2 & 2 & -2 \\
-2 & 2 & 2 & -2 & 2 & -2 & -2 & 2 \\
2 & -2 & -2 & 2 & -2 & 2 & 2 & -2 \\
2 & -2 & -2 & 2 & -2 & 2 & 2 & -2 \\
-2 & 2 & 2 & -2 & 2 & -2 & -2 & 2
\end{array}\right],
$$

where the diagrams are ordered as shown in figure 20.

\section{A.2 $(1,1,1, \ldots 1, n)$ webs}

The $n=2$ case can be obtained by relabelling external lines in the $(1,2,1)$ web shown in figure 1 , whose mixing matrix is given in eq. (A.1). The $n=3$ case is shown in figure 21, and the mixing matrix is

$$
R_{(1,1,1,3)}=\frac{1}{6}\left[\begin{array}{rrrrrr}
2 & -1 & -1 & 2 & -1 & -1 \\
-1 & 2 & -1 & -1 & -1 & 2 \\
-1 & -1 & 2 & -1 & 2 & -1 \\
2 & -1 & -1 & 2 & -1 & -1 \\
-1 & -1 & 2 & -1 & 2 & -1 \\
-1 & 2 & -1 & -1 & -1 & 2
\end{array}\right],
$$

with diagrams ordered as in the figure. 
For the $n=4$ case $(1,1,1,1,4)$, we do not reproduce the 24 web diagrams here. We may label each diagram concisely by a permutation of (1234), where this denotes the ordering of the gluons on the parton line with 4 attachments (away from the hard interaction), such that gluon $i$ joins this line with external line $i$. One then has the mixing matrix

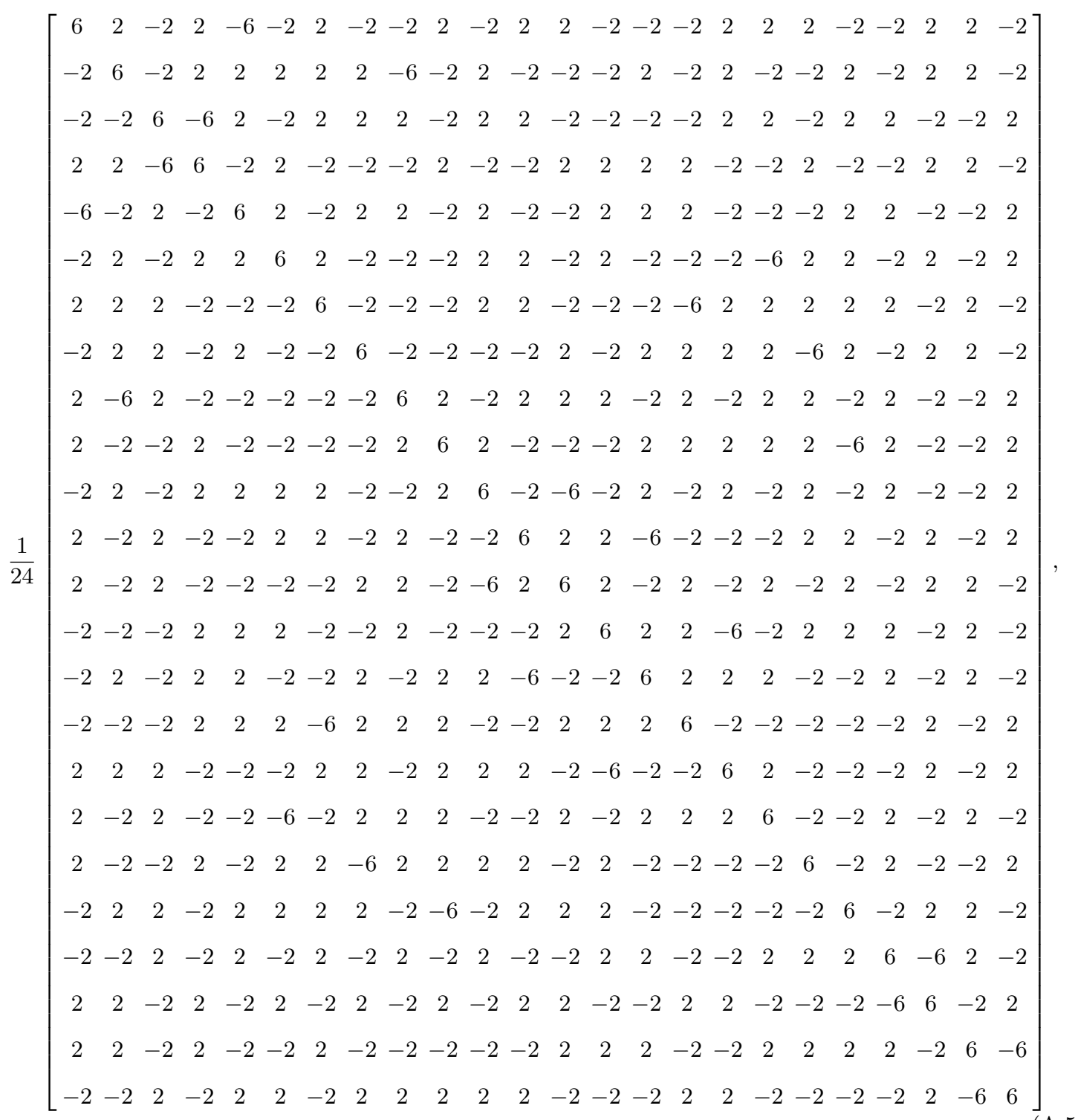

where the ordering of the diagrams is given by

(2314, 1243, 3124, 4213, 4132, 3142, 4321, 4231, 3421, 4312, 2341, 4123, 1432, 2431, 3214, 1234, $1342,2413,1324,2134,1423,3241,3412,2143)$.

\section{B Eulerian numbers}

In this appendix, we briefly summarise the properties of Eulerian numbers, which occur in the solution of the web-mixing matrix for $(1,1, \ldots, 1, n)$ webs. 


\begin{tabular}{|cc|ccccccc|}
\hline & & & \multicolumn{1}{|c|}{$r$} & & & \\
& & 0 & 1 & 2 & 3 & 4 & 5 & 6 \\
\hline & 1 & 1 & & & & & & \\
& 2 & 1 & 1 & & & & & \\
& 3 & 1 & 4 & 1 & & & & \\
$n$ & 4 & 1 & 11 & 11 & 1 & & & \\
& 5 & 1 & 26 & 66 & 26 & 1 & & \\
& 6 & 1 & 57 & 302 & 302 & 57 & 1 & \\
& 7 & 1 & 120 & 1191 & 2416 & 1191 & 120 & 1 \\
\hline
\end{tabular}

Table 2. Table of Eulerian numbers.

The Eulerian number $\left\langle\begin{array}{l}n \\ r\end{array}\right\rangle$ counts the number of permutations of $n$ objects with $r$ ascents (consecutive pairs of rising numbers), where $0 \leq r \leq n-1$. Results for the first few values of $n$ and $r$ are shown in table 2 .

A number of identities regarding Eulerian numbers are known. For the present paper, the following identity is useful:

$$
\sum_{r=0}^{n-1}(-1)^{r}\left(\begin{array}{c}
n-1 \\
r
\end{array}\right)^{-1}\left\langle\begin{array}{l}
n \\
r
\end{array}\right\rangle=0 .
$$

\section{Results for $s(D)$ for $(1,2,2, \ldots, 2,1)$ webs}

In this appendix, we gather results for the $s(D)$ factors for $(1,2,2, \ldots 1)$ webs, up to nine loop order. These are given in table 3, which also lists the multiplicities of each $s(D)$ values. One may check explicitly in each case that the total number of values is equal to $2^{n-1}$ (the total number of diagrams). Note that a closed form solution for these numbers (in terms of known combinatorial functions) is not currently possible. It is clear from studying table 3 that there are a number of interesting relationships. For example, the multiplicities of each $s(D)$ value are always even. This follows from the fact that the $s(D)$ value for a given web diagram $D$ must be the same as its conjugate diagram $D^{*}$, as well as for the Hasse diagram obtained by reflecting the Hasse diagram of $D$ about the vertical (by symmetry). The highest $s(D)$ value in each case corresponds to the Hasse diagrams which are maximally kinked. Such posets are referred to as zig-zag posets or fences in the mathematical literature, such that the highest $s(D)$ value for any given $n$ is the number of linear extensions of the zig-zag poset. 


\begin{tabular}{|c|c|c|c|c|c|c|c|}
\hline Number of Gluons & $s(D)$ & Multiplicity & & & & & \\
\hline$n=1$ & 1 & 1 & & 85 & 4 & 461 & 4 \\
\hline$n=2$ & 1 & 2 & & 99 & 4 & 470 & 4 \\
\hline \multirow{2}{*}{$n=3$} & 1 & 2 & & 105 & 4 & 496 & 4 \\
\hline & 2 & 2 & & 125 & 4 & 512 & 4 \\
\hline \multirow[t]{3}{*}{$n=4$} & 1 & 2 & & 133 & 4 & 595 & 4 \\
\hline & 3 & 4 & & 155 & 4 & 632 & 4 \\
\hline & 5 & 2 & & 181 & 2 & 664 & 4 \\
\hline \multirow[t]{6}{*}{$n=5$} & 1 & 2 & & 189 & 4 & 685 & 2 \\
\hline & 4 & 4 & & 203 & 4 & 728 & 4 \\
\hline & 6 & 2 & & 217 & 4 & 784 & 4 \\
\hline & 9 & 4 & & 245 & 4 & 785 & 4 \\
\hline & 11 & 2 & & 259 & 4 & 812 & 4 \\
\hline & 16 & 2 & & 315 & 8 & 880 & 2 \\
\hline \multirow[t]{9}{*}{$n=6$} & 1 & 2 & & 323 & 4 & 1016 & 4 \\
\hline & 5 & 4 & & 365 & 2 & 1051 & 4 \\
\hline & 10 & 4 & & 407 & 4 & 1099 & 4 \\
\hline & 14 & 4 & & 413 & 4 & 1100 & 4 \\
\hline & 19 & 4 & & 449 & 2 & 1141 & 4 \\
\hline & 26 & 4 & & 477 & 4 & 1168 & 4 \\
\hline & 35 & 4 & & 531 & 4 & 1253 & 4 \\
\hline & 40 & 4 & & 573 & 4 & 1351 & 4 \\
\hline & 61 & 2 & & 589 & 2 & 1421 & 4 \\
\hline \multirow[t]{19}{*}{$n=7$} & 1 & 2 & & 643 & 4 & 1456 & 2 \\
\hline & 6 & 4 & & 791 & 4 & 1457 & 4 \\
\hline & 15 & 4 & & 875 & 4 & 1513 & 2 \\
\hline & 20 & 6 & & 917 & 4 & 1519 & 4 \\
\hline & 29 & 2 & & 1385 & 2 & 1667 & 4 \\
\hline & 34 & 4 & $n=9$ & 1 & 2 & 1735 & 4 \\
\hline & 50 & 4 & & 8 & 4 & 1856 & 4 \\
\hline & 55 & 4 & & 28 & 4 & 1889 & 4 \\
\hline & 64 & 4 & & 35 & 4 & 2051 & 4 \\
\hline & 71 & 2 & & 55 & 2 & 2107 & 4 \\
\hline & 78 & 4 & & 56 & 4 & 2144 & 4 \\
\hline & 90 & 2 & & 70 & 2 & 2261 & 4 \\
\hline & 99 & 4 & & 83 & 4 & 2312 & 4 \\
\hline & 111 & 4 & & 125 & 4 & 2590 & 4 \\
\hline & 132 & 2 & & 133 & 4 & 2701 & 2 \\
\hline & 155 & 4 & & 160 & 4 & 2780 & 2 \\
\hline & 169 & 2 & & 161 & 4 & 2890 & 4 \\
\hline & 181 & 4 & & 208 & 4 & 2990 & 4 \\
\hline & 272 & 2 & & 245 & 4 & 3194 & 4 \\
\hline \multirow[t]{8}{*}{$n=8$} & 1 & 2 & & 245 & 4 & 3268 & 4 \\
\hline & 7 & 4 & & 259 & 4 & 3526 & 4 \\
\hline & 21 & 4 & & 295 & 4 & 3736 & 4 \\
\hline & 27 & 4 & & 350 & 4 & 4529 & 4 \\
\hline & 35 & 4 & & 370 & 4 & 4985 & 4 \\
\hline & 41 & 2 & & 379 & 4 & 5095 & 2 \\
\hline & 55 & 4 & & 412 & 4 & 5263 & 4 \\
\hline & 69 & 2 & & 448 & 2 & 7936 & 2 \\
\hline
\end{tabular}

Table 3. $s(D)$ values for the $(1,2,2, \ldots 1)$ web, where $n$ is the number of gluon exchanges. Also shown is the multiplicity of each $s(D)$ value. 
Open Access. This article is distributed under the terms of the Creative Commons Attribution License (CC-BY 4.0), which permits any use, distribution and reproduction in any medium, provided the original author(s) and source are credited.

\section{References}

[1] I.Y. Arefeva, Quantum contour field equations, Phys. Lett. B 93 (1980) 347 [INSPIRE].

[2] A.M. Polyakov, Gauge fields as rings of glue, Nucl. Phys. B 164 (1980) 171 [INSPIRE].

[3] V. Dotsenko and S. Vergeles, Renormalizability of phase factors in the non-Abelian gauge theory, Nucl. Phys. B 169 (1980) 527 [InSPIRE].

[4] R.A. Brandt, F. Neri and M.-A. Sato, Renormalization of loop functions for all loops, Phys. Rev. D 24 (1981) 879 [INSPIRE].

[5] G. Korchemsky and A. Radyushkin, Loop space formalism and renormalization group for the infrared asymptotics of QCD, Phys. Lett. B 171 (1986) 459 [INSPIRE].

[6] S. Ivanov, G. Korchemsky and A. Radyushkin, Infrared asymptotics of perturbative QCD: contour gauges, Sov. J. Nucl. Phys. 44 (1986) 145 [Yad. Fiz. 44 (1986) 230] [InSPIRE].

[7] G. Korchemsky and A. Radyushkin, Infrared asymptotics of perturbative QCD: renormalization properties of the Wilson loops in higher orders of perturbation theory, Sov. J. Nucl. Phys. 44 (1986) 877 [Yad. Fiz. 44 (1986) 1351] [inSPIRE].

[8] G. Korchemsky and A. Radyushkin, Infrared asymptotics of perturbative QCD. Quark and gluon propagators, Sov. J. Nucl. Phys. 45 (1987) 127 [Yad. Fiz. 45 (1987) 198] [INSPIRE].

[9] G. Korchemsky and A. Radyushkin, Infrared asymptotics of perturbative QCD. Vertex functions, Sov. J. Nucl. Phys. 45 (1987) 910 [Yad. Fiz. 45 (1987) 1466] [inSPIRE].

[10] G. Korchemsky and A. Radyushkin, Renormalization of the Wilson loops beyond the leading order, Nucl. Phys. B 283 (1987) 342 [InSPIRE].

[11] G. Korchemsky, Sudakov form-factor in QCD, Phys. Lett. B 220 (1989) 629 [INSPIRE].

[12] G. Korchemsky, Asymptotics of the Altarelli-Parisi-Lipatov evolution kernels of parton distributions, Mod. Phys. Lett. A 4 (1989) 1257 [InSPIRE].

[13] J.C. Collins, Sudakov form-factors, Adv. Ser. Direct. High Energy Phys. 5 (1989) 573 [hep-ph/0312336] [INSPIRE].

[14] G. Korchemsky and A. Radyushkin, Infrared factorization, Wilson lines and the heavy quark limit, Phys. Lett. B 279 (1992) 359 [hep-ph/9203222] [INSPIRE].

[15] N. Kidonakis, G. Oderda and G.F. Sterman, Evolution of color exchange in QCD hard scattering, Nucl. Phys. B 531 (1998) 365 [hep-ph/9803241] [INSPIRE].

[16] N. Kidonakis and G.F. Sterman, Resummation for QCD hard scattering, Nucl. Phys. B 505 (1997) 321 [hep-ph/9705234] [INSPIRE].

[17] N. Kidonakis and G.F. Sterman, Subleading logarithms in QCD hard scattering, Phys. Lett. B 387 (1996) 867 [INSPIRE].

[18] N. Kidonakis, Next-to-next-to-leading soft-gluon corrections for the top quark cross section and transverse momentum distribution, Phys. Rev. D 82 (2010) 114030 [arXiv:1009.4935] [INSPIRE]. 
[19] J. Drummond, J. Henn, G. Korchemsky and E. Sokatchev, On planar gluon amplitudes/Wilson loops duality, Nucl. Phys. B 795 (2008) 52 [arXiv:0709.2368] [InSPIRE].

[20] B. Basso, G. Korchemsky and J. Kotanski, Cusp anomalous dimension in maximally supersymmetric Yang-Mills theory at strong coupling, Phys. Rev. Lett. 100 (2008) 091601 [arXiv: 0708.3933] [INSPIRE].

[21] L.F. Alday and J.M. Maldacena, Gluon scattering amplitudes at strong coupling, JHEP 06 (2007) 064 [arXiv:0705.0303] [INSPIRE].

[22] V. Pestun, Localization of gauge theory on a four-sphere and supersymmetric Wilson loops, Commun. Math. Phys. 313 (2012) 71 [arXiv:0712.2824] [InSPIRE].

[23] N. Drukker, Integrable Wilson loops, JHEP 10 (2013) 135 [arXiv:1203.1617] [INSPIRE].

[24] Y.-T. Chien, M.D. Schwartz, D. Simmons-Duffin and I.W. Stewart, Jet physics from static charges in AdS, Phys. Rev. D 85 (2012) 045010 [arXiv:1109.6010] [InSPIRE].

[25] I. Cherednikov, T. Mertens and F. Van der Veken, Cusped light-like Wilson loops in gauge theories, Phys. Part. Nucl. 44 (2013) 250 [arXiv:1210.1767] [INSPIRE].

[26] I. Cherednikov, T. Mertens and F. Van der Veken, Evolution of cusped light-like Wilson loops and geometry of the loop space, Phys. Rev. D 86 (2012) 085035 [arXiv:1208.1631] [INSPIRE].

[27] J.M. Henn and T. Huber, The four-loop cusp anomalous dimension in $\mathcal{N}=4$ super Yang-Mills and analytic integration techniques for Wilson line integrals, JHEP 09 (2013) 147 [arXiv: 1304.6418] [INSPIRE].

[28] S.G. Naculich and H.J. Schnitzer, Eikonal methods applied to gravitational scattering amplitudes, JHEP 05 (2011) 087 [arXiv:1101.1524] [INSPIRE].

[29] C.D. White, Factorization properties of soft graviton amplitudes, JHEP 05 (2011) 060 [arXiv: 1103.2981] [INSPIRE].

[30] R. Akhoury, R. Saotome and G. Sterman, Collinear and soft divergences in perturbative quantum gravity, Phys. Rev. D 84 (2011) 104040 [arXiv:1109.0270] [INSPIRE].

[31] D. Miller and C. White, The gravitational cusp anomalous dimension from AdS space, Phys. Rev. D 85 (2012) 104034 [arXiv:1201.2358] [INSPIRE].

[32] M. Beneke and G. Kirilin, Soft-collinear gravity, JHEP 09 (2012) 066 [arXiv:1207.4926] [INSPIRE].

[33] E. Gardi and L. Magnea, Infrared singularities in QCD amplitudes, Nuovo Cim. C 32 (2009) 137 [arXiv:0908.3273] [INSPIRE].

[34] J.C. Collins, D.E. Soper and G.F. Sterman, Factorization of hard processes in QCD, Adv. Ser. Direct. High Energy Phys. 5 (1988) 1 [hep-ph/0409313] [INSPIRE].

[35] G. Korchemsky and G. Marchesini, Structure function for large $x$ and renormalization of Wilson loop, Nucl. Phys. B 406 (1993) 225 [hep-ph/9210281] [INSPIRE].

[36] G. Korchemsky and G. Marchesini, Resummation of large infrared corrections using Wilson loops, Phys. Lett. B 313 (1993) 433 [InSPIRE].

[37] S. Catani, M.L. Mangano, P. Nason and L. Trentadue, The resummation of soft gluons in hadronic collisions, Nucl. Phys. B 478 (1996) 273 [hep-ph/9604351] [INSPIRE]. 
[38] G. Oderda, Dijet rapidity gaps in photoproduction from perturbative QCD, Phys. Rev. D 61 (2000) 014004 [hep-ph/9903240] [INSPIRE].

[39] R. Bonciani, S. Catani, M.L. Mangano and P. Nason, NLL resummation of the heavy quark hadroproduction cross-section, Nucl. Phys. B 529 (1998) 424 [Erratum ibid. B 803 (2008) 234] [hep-ph/9801375] [INSPIRE].

[40] M. Beneke, P. Falgari and C. Schwinn, Soft radiation in heavy-particle pair production: all-order colour structure and two-loop anomalous dimension, Nucl. Phys. B 828 (2010) 69 [arXiv: 0907.1443] [inSPIRE].

[41] M. Beneke, M. Czakon, P. Falgari, A. Mitov and C. Schwinn, Threshold expansion of the $g g(q \bar{q}) \rightarrow Q \bar{Q}+X$ cross section at $\mathcal{O}\left(\alpha_{s}^{4}\right)$, Phys. Lett. B $690(2010) 483$ [arXiv:0911.5166] [INSPIRE].

[42] V. Ahrens, A. Ferroglia, M. Neubert, B.D. Pecjak and L.L. Yang, Renormalization-group improved predictions for top-quark pair production at hadron colliders, JHEP 09 (2010) 097 [arXiv: 1003.5827] [INSPIRE].

[43] M. Czakon, P. Fiedler and A. Mitov, The total top quark pair production cross-section at hadron colliders through $\mathcal{O}\left(\alpha_{S}^{4}\right)$, Phys. Rev. Lett. 110 (2013) 252004 [arXiv:1303.6254] [INSPIRE].

[44] C.W. Bauer, S. Fleming and M.E. Luke, Summing Sudakov logarithms in $B \rightarrow X(s \gamma)$ in effective field theory, Phys. Rev. D 63 (2000) 014006 [hep-ph/0005275] [InSPIRE].

[45] C.W. Bauer, S. Fleming, D. Pirjol and I.W. Stewart, An effective field theory for collinear and soft gluons: heavy to light decays, Phys. Rev. D 63 (2001) 114020 [hep-ph/0011336] [INSPIRE].

[46] C.W. Bauer and I.W. Stewart, Invariant operators in collinear effective theory, Phys. Lett. B 516 (2001) 134 [hep-ph/0107001] [INSPIRE].

[47] C.W. Bauer, D. Pirjol and I.W. Stewart, Soft collinear factorization in effective field theory, Phys. Rev. D 65 (2002) 054022 [hep-ph/0109045] [INSPIRE].

[48] C.W. Bauer, S. Fleming, D. Pirjol, I.Z. Rothstein and I.W. Stewart, Hard scattering factorization from effective field theory, Phys. Rev. D 66 (2002) 014017 [hep-ph/0202088] [INSPIRE].

[49] T. Becher and M. Neubert, Threshold resummation in momentum space from effective field theory, Phys. Rev. Lett. 97 (2006) 082001 [hep-ph/0605050] [INSPIRE].

[50] T. Becher, M. Neubert and B.D. Pecjak, Factorization and momentum-space resummation in deep-inelastic scattering, JHEP 01 (2007) 076 [hep-ph/0607228] [INSPIRE].

[51] T. Becher, M. Neubert and G. Xu, Dynamical threshold enhancement and resummation in Drell-Yan production, JHEP 07 (2008) 030 [arXiv:0710.0680] [INSPIRE].

[52] D. Yennie, S.C. Frautschi and H. Suura, The infrared divergence phenomena and high-energy processes, Annals Phys. 13 (1961) 379 [INSPIRE].

[53] G.F. Sterman, Summation of large corrections to short distance hadronic cross-sections, Nucl. Phys. B 281 (1987) 310 [InSPIRE].

[54] S. Catani and L. Trentadue, Resummation of the QCD perturbative series for hard processes, Nucl. Phys. B 327 (1989) 323 [INSPIRE]. 
[55] E. Laenen, G. Stavenga and C.D. White, Path integral approach to eikonal and next-to-eikonal exponentiation, JHEP 03 (2009) 054 [arXiv:0811.2067] [INSPIRE].

[56] M.G. Sotiropoulos and G.F. Sterman, Color exchange in near forward hard elastic scattering, Nucl. Phys. B 419 (1994) 59 [hep-ph/9310279] [INSPIRE].

[57] G.P. Korchemsky, On near forward high-energy scattering in QCD, Phys. Lett. B 325 (1994) 459 [hep-ph/9311294] [INSPIRE].

[58] I. Korchemskaya and G. Korchemsky, High-energy scattering in QCD and cross singularities of Wilson loops, Nucl. Phys. B 437 (1995) 127 [hep-ph/9409446] [INSPIRE].

[59] I. Korchemskaya and G. Korchemsky, Evolution equation for gluon Regge trajectory, Phys. Lett. B 387 (1996) 346 [hep-ph/9607229] [INSPIRE].

[60] I. Balitsky, Operator expansion for high-energy scattering, Nucl. Phys. B 463 (1996) 99 [hep-ph/9509348] [INSPIRE].

[61] Y.V. Kovchegov, Non-Abelian Weizsacker-Williams field and a two-dimensional effective color charge density for a very large nucleus, Phys. Rev. D 54 (1996) 5463 [hep-ph/9605446] [INSPIRE].

[62] I. Balitsky, High-energy QCD and Wilson lines, hep-ph/0101042 [INSPIRE].

[63] I. Balitsky and G.A. Chirilli, High-energy amplitudes in $N=4$ SYM in the next-to-leading order, Phys. Lett. B 687 (2010) 204 [arXiv:0911.5192] [INSPIRE].

[64] J. Jalilian-Marian, A. Kovner, L.D. McLerran and H. Weigert, The intrinsic glue distribution at very small $x$, Phys. Rev. D 55 (1997) 5414 [hep-ph/9606337] [INSPIRE].

[65] E. Gardi, J. Kuokkanen, K. Rummukainen and H. Weigert, Running coupling and power corrections in nonlinear evolution at the high-energy limit, Nucl. Phys. A 784 (2007) 282 [hep-ph/0609087] [INSPIRE].

[66] V. Del Duca, C. Duhr, E. Gardi, L. Magnea and C.D. White, An infrared approach to Reggeization, Phys. Rev. D 85 (2012) 071104 [arXiv:1108.5947] [INSPIRE].

[67] V. Del Duca, C. Duhr, E. Gardi, L. Magnea and C.D. White, The infrared structure of gauge theory amplitudes in the high-energy limit, JHEP 12 (2011) 021 [arXiv:1109.3581] [INSPIRE].

[68] Y.V. Kovchegov and E. Levin, Quantum chromodynamics at high energy, Cambridge Univ. Pr., Cambridge U.K. (2012) [INSPIRE].

[69] A.H. Mueller, Soft gluons in the infinite momentum wave function and the BFKL Pomeron, Nucl. Phys. B 415 (1994) 373 [InSPIRE].

[70] S. Melville, S. Naculich, H. Schnitzer and C. White, Wilson line approach to gravity in the high energy limit, arXiv:1306.6019 [INSPIRE].

[71] R. Akhoury, R. Saotome and G. Sterman, High energy scattering in perturbative quantum gravity at next to leading power, arXiv:1308.5204 [INSPIRE].

[72] J. Ware, R. Saotome and R. Akhoury, Construction of an asymptotic $S$ matrix for perturbative quantum gravity, JHEP 10 (2013) 159 [arXiv:1308.6285] [INSPIRE].

[73] J. Gatheral, Exponentiation of eikonal cross-sections in non-Abelian gauge theories, Phys. Lett. B 133 (1983) 90 [INSPIRE]. 
[74] J. Frenkel and J. Taylor, Non-Abelian eikonal exponentiation, Nucl. Phys. B 246 (1984) 231 [INSPIRE].

[75] G.F. Sterman, Infrared divergences in perturbative QCD (talk), AIP Conf. Proc. 74 (1981) 22 [INSPIRE].

[76] S.M. Aybat, L.J. Dixon and G.F. Sterman, The two-loop anomalous dimension matrix for soft gluon exchange, Phys. Rev. Lett. 97 (2006) 072001 [hep-ph/0606254] [INSPIRE].

[77] S.M. Aybat, L.J. Dixon and G.F. Sterman, The two-loop soft anomalous dimension matrix and resummation at next-to-next-to leading pole, Phys. Rev. D 74 (2006) 074004 [hep-ph/0607309] [INSPIRE].

[78] A. Ferroglia, M. Neubert, B.D. Pecjak and L.L. Yang, Two-loop divergences of scattering amplitudes with massive partons, Phys. Rev. Lett. 103 (2009) 201601 [arXiv:0907.4791] [INSPIRE].

[79] A. Ferroglia, M. Neubert, B.D. Pecjak and L.L. Yang, Two-loop divergences of massive scattering amplitudes in non-Abelian gauge theories, JHEP 11 (2009) 062 [arXiv:0908.3676] [INSPIRE].

[80] A. Mitov, G.F. Sterman and I. Sung, Computation of the soft anomalous dimension matrix in coordinate space, Phys. Rev. D 82 (2010) 034020 [arXiv: 1005.4646] [InSPIRE].

[81] T. Becher and M. Neubert, Infrared singularities of scattering amplitudes in perturbative QCD, Phys. Rev. Lett. 102 (2009) 162001 [arXiv:0901.0722] [INSPIRE].

[82] T. Becher and M. Neubert, On the structure of infrared singularities of gauge-theory amplitudes, JHEP 06 (2009) 081 [Erratum ibid. 11 (2013) 024] [arXiv:0903.1126] [inSPIRE].

[83] E. Gardi and L. Magnea, Factorization constraints for soft anomalous dimensions in QCD scattering amplitudes, JHEP 03 (2009) 079 [arXiv:0901.1091] [INSPIRE].

[84] L.J. Dixon, E. Gardi and L. Magnea, On soft singularities at three loops and beyond, JHEP 02 (2010) 081 [arXiv: 0910.3653] [INSPIRE].

[85] V. Ahrens, M. Neubert and L. Vernazza, Structure of infrared singularities of gauge-theory amplitudes at three and four loops, JHEP 09 (2012) 138 [arXiv:1208.4847] [INSPIRE].

[86] S.G. Naculich, H. Nastase and H.J. Schnitzer, All-loop infrared-divergent behavior of most-subleading-color gauge-theory amplitudes, JHEP 04 (2013) 114 [arXiv:1301.2234] [INSPIRE].

[87] S. Caron-Huot, When does the gluon reggeize?, arXiv:1309.6521 [INSPIRE].

[88] E. Gardi, E. Laenen, G. Stavenga and C.D. White, Webs in multiparton scattering using the replica trick, JHEP 11 (2010) 155 [arXiv:1008.0098] [INSPIRE].

[89] E. Gardi and C.D. White, General properties of multiparton webs: proofs from combinatorics, JHEP 03 (2011) 079 [arXiv:1102.0756] [INSPIRE].

[90] E. Gardi, J.M. Smillie and C.D. White, On the renormalization of multiparton webs, JHEP 09 (2011) 114 [arXiv:1108.1357] [INSPIRE].

[91] E. Gardi, J.M. Smillie and C.D. White, The non-Abelian exponentiation theorem for multiple Wilson lines, JHEP 06 (2013) 088 [arXiv:1304.7040] [INSPIRE].

[92] A. Mitov, G. Sterman and I. Sung, Diagrammatic exponentiation for products of Wilson lines, Phys. Rev. D 82 (2010) 096010 [arXiv: 1008.0099] [InSPIRE]. 
[93] E. Gardi, From webs to polylogarithms, arXiv:1310.5268 [INSPIRE].

[94] G. Falcioni, E. Gardi, M. Harley, L. Magnea and C.D. White, Calculating three-loop webs, in preparation.

[95] M. Dukes, E. Gardi, E. Steingrimsson and C.D. White, Web worlds, web-colouring matrices and web-mixing matrices, J. Comb. Theory Ser. A 120 (2013) 1012 [arXiv:1301.6576] [INSPIRE].

[96] V. Del Duca, L.J. Dixon and F. Maltoni, New color decompositions for gauge amplitudes at tree and loop level, Nucl. Phys. B 571 (2000) 51 [hep-ph/9910563] [INSPIRE].

[97] D.R. Mazur, Combinatorics: a guided tour, Mathematical Association of America, U.S.A. (2010).

[98] G. Brightwell and P. Winkler, Counting linear extensions, Order 8 (1991) 225.

[99] B. Sury, T. Wang and F.Z. Zhao, Identities involving reciprocals of binomial coefficients, J. Integer Seq. 7 (2004) 04.2.8. 